\title{
Asymptotic Analysis of Heat Transfer in Composite Materials with Nonlinear Thermal Properties
}

\author{
Igor V. Andrianov ${ }^{\mathrm{a}}$, Heiko Topol ${ }^{\mathrm{b}}$, Vladyslav V. Danishevskyy ${ }^{\mathrm{c}}$ \\ ${ }^{a}$ Institute of General Mechanics, RWTH Aachen University, Templergraben 64, 52062 Aachen, Germany \\ ${ }^{b}$ Center for Advanced Materials, Qatar University, P.O. Box 2713, Doha, Qatar \\ ${ }^{c}$ School of Computing and Mathematics, Keele University, Staffordshire, ST5 5BG, UK
}

\begin{abstract}
We study heat transfer through a composite with periodic microstructure. The thermal conductivity of the constituents is assumed to be temperature-dependent, and it is modeled as a polynomial in terms of the temperature. The thermal resistance between the constituents is taken to be nonlinear. In order to determine the effective thermal properties of the material, we apply the asymptotic homogenization method. We discuss different approaches to determine these effective properties for the different volume fractions of the inclusions. For high volume fractions of the inclusion, we apply the lubrication theory. In the case of low volume fractions of the inclusions, we apply the three-phase model. Comparing some special cases of our results to existing ones in the literature shows a good accuracy.
\end{abstract}

Keywords: heat transfer, composites, nonlinearity, asymptotic homogenizaton method, three-phase model, lubrication theory

\section{Introduction}

Modeling of the thermal properties of composites might be challenging, especially when the size of the heterogeneities is significantly smaller than the macroscopic size of the considered structure. In order to simplify the treatment of heat diffusion problems, different approaches have been developed, in which the original heterogeneous material is replaced by a homogenized or effective material with the same macroscopic properties as the original heterogeneous material. Early works on this topic are, for example, the works of Hershey [1], Hill [2], Kerner [3], Kröner [4], Keller [5], and van der Poel [6]. Examples for works on computational homogenization are article of Özdemir et al. [7], and the work of Geers et al. [8] discusses some trends and challenges in this field.

A powerful and wide-spread technique denoted as the asymptotic homogenization method (AHM) has been developed in order to obtain the effective properties of different asymptotic orders of heterogeneous materials with periodic microstructures. The theory behind this technique is described, for example, in the books of Bensoussan et al. [9] and Panasenko [10]. The AHM allows to investigate a macroscopic boundary value problem within a single repeated unit cell of the microstructure. In this approach, a small parameter is introduced, which relates the size of the heterogeneities to the size of the macroscopic problem. The original coordinate variables are then replaced by so-called fast coordinate variables, which consider the problem on the micro-scale, and by slow coordinates, which consider the problem on the macro-scale. The AHM has been Preprint submitted to International Journal of Heat and Mass Transfer 
applied to analyze different types of homogenization problem, for example to investigate wave propagation in fiber-reinforced composites $[11,12]$. There also exist numerous articles, which have applied the AHM to determine the effective thermal properties of composites, for example, Allaire [13] and Zhang et al. [14]. Telega et al. [15] applied the AHM to study heat transfer, which is formulated as a minimization problem. Gałka et al. [16] took temperature-dependent thermal parameters of the constituents in the homogenization procedure into account. Allaire \& Habibi [17] and Yang et al. [18] analyze heat transfer in porous materials, and they include conduction, convection, and radiation into their considerations. A popular method to investigate the effective properties of composites with a low volume fraction of the inclusion phase is denoted as the three-phase model [19]. The application of such model for the AHM has been discussed and justified in [20]. If the volume fraction of the inclusions approach its maximum, then the close packing model [21], also denoted as the lubrication theory, has been applied in different works. A broader review of trends of the application of the AHM to obtain the effective properties of composites is provided by Kalamkarov et al. [22], who state that the different developed methods reveal their strengths and disadvantages, and therefore these methods have to be treated as complementary tools.

The effective macroscopic properties result from the properties and the distribution of the constituents, but also from the interaction of the constituents. Composites might reveal thermal resistance between the different constituents, which might for example result from imperfect contact, cracks, or from an interphase material. An early work on thermal interfacial resistance is the article of Kapitza [23]. Examples for composites with coated inclusions is micro-encapsulated paraffin-spheres, which has been studied in different experiments on thermal regulations of buildings (Karkri et al. [24, 25]). Theoretical modeling works on the effective thermal properties, which consider such resistance, are, for example, Quang et al. [26, 27, 28] and Andrianov et al. [29]. There exist different interface models which have been taken into account in different studies, such as hybrid interphase regions [30], and inhomogeneous interphases [31].

Our article is organized as follows: In Sec. 2 we introduce the herein considered boundary value problem, the applied heat diffusion model, and thermal resistance models. In Sec. 3 we discuss the application of the AHM in order to obtain the effective thermal parameters of the considered composite. The case of large volume fractions of the inclusion is discussed in detail in Sec. 4, as well as the case of a layered composite. Illustrative examples are introduced to discuss the different features of the derived heat propagation models. In Sec. 5 we apply the three-phase model for composites with low volume fractions of the inclusions, and we discuss the cases of parallel fibers and spherical inclusions in the matrix. Special cases of our results are compared to known results from the literature. In the final section, we discuss the obtained results, and we provide a brief outlook.

\section{Nonlinear heat diffusion in a composite}

Consider a heterogeneous material with a periodic microstructure, which is assumed to consist of two constituents, the inclusion $\Omega^{(1)}$ and the surrounding matrix $\Omega^{(2)}$. In the framework of this article we will mainly focus on inclusions of spherical shape, as shown in Fig. 1, and on inclusions of cylindrical shape. In a Cartesian coordinate system with the three base unit vectors $\left\{\mathbf{E}_{1}, \mathbf{E}_{2}, \mathbf{E}_{3}\right\}$, the microstructure of the material can be described by repeated unit cells in form of parallelepipeds of the lengths $\ell_{k}$ in the $\mathbf{E}_{k}$-directions, $k=1,2,3$. The volume of such unit cell then becomes $v=\ell_{1} \ell_{2} \ell_{3}$. In the following, we want to study heat diffusion in such composite. Section 2.1 gives a brief general summary on the applied heat diffusion model, and Sec. 2.2 


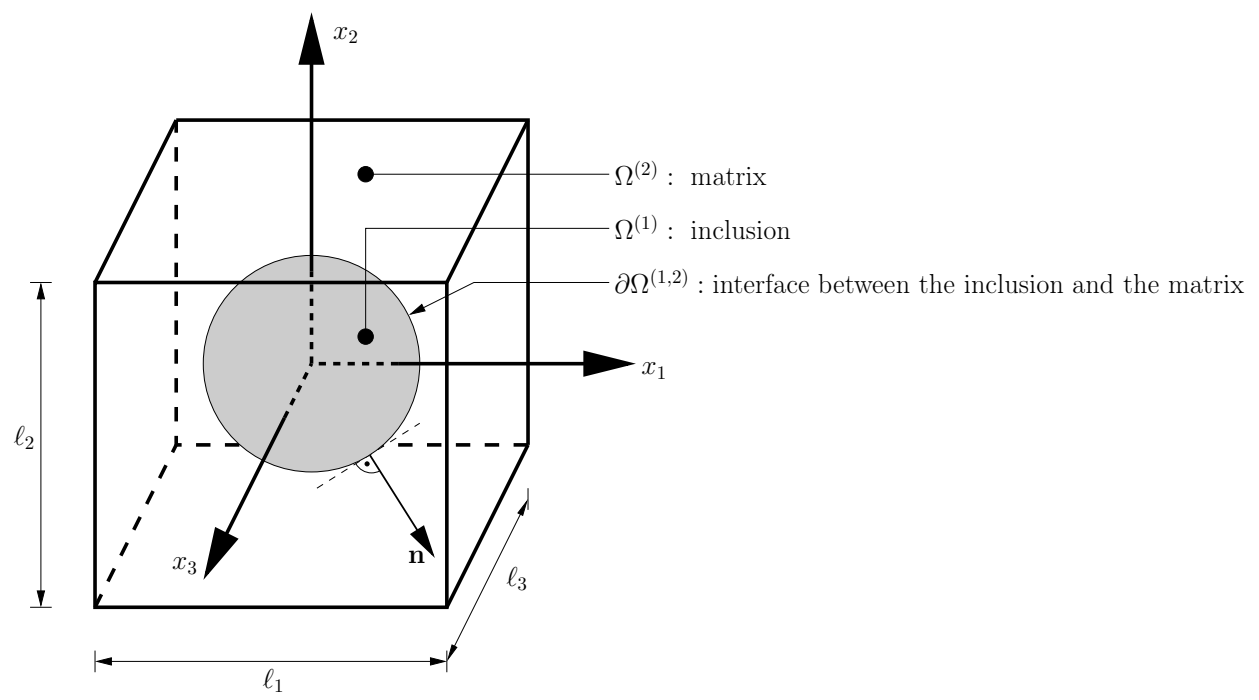

Figure 1: A single unit cell of the periodic composite microstructure: The inclusion $\Omega^{(1)}$ is surrounded by the matrix $\Omega^{(2)}$. The interface between $\Omega^{(1)}$ and $\Omega^{(2)}$ is denoted as $\partial \Omega^{(1,2)}$, and $\mathbf{n}$ is the outer normal unit vector to the inclusion.

\subsection{Summary of the heat equation model}

$$
q(T(\mathbf{x}, t))=-\kappa(T(\mathbf{x}, t)) \frac{\partial T(\mathbf{x}, t)}{\partial x_{k}}, \quad k=1,2,3,
$$

70

where $\kappa=\kappa(T(\mathbf{x}, t))$ is the thermal conductivity and $T=T(\mathbf{x}, t)$ is the temperature at the location

$$
\mathbf{x}=\mathbf{E}_{1} x_{1}+\mathbf{E}_{2} x_{2}+\mathbf{E}_{3} x_{3}
$$

at time $t$. Note that (1) represent a form of the heat flux equation in which the thermal properties are taken to be independent from the considered direction. We model the thermal conductivity $\kappa=\kappa(T(\mathbf{x}, t))$ as a function of the temperature, and therefore it is taken to be a polynomial in terms of the temperature in the form

$$
\kappa(T(\mathbf{x}, t))=\sum_{i=0}^{i_{\max }} a_{i}[T(\mathbf{x}, t)]^{i}=a_{0}+a_{1} T(\mathbf{x}, t)+\ldots,
$$

75 where $a_{i}$ are constants. Such model has been applied, for example, by Lienemann et al. [32], and

76 this general form allows to describe different types of effects: The first term of the right side of (3)

77 is the linear term, which is independent from the temperature. The following higher-order terms

78 define the temperature-dependence of the conductivity. The number of terms in (3) depends 
on the accuracy of the conductivity model in the considered temperature range. If for example the considered temperature range is low, then it might be sufficient to restrict (3) to the leading term. On a large temperature range the thermal conductivity might reveal a strong nonlinear behavior. To give an example, on the temperature range from 0 Kelvin to its melting point, the conductivity of aluminum strongly increases to its maximum, and then slowly decreases with rising temperatures (see, for example, Table 3a in Hatch [33]). Thermal conductivities in the form $\kappa=a_{m} T^{m}, m=2,3, \ldots$ have been studies in different works, and for an overview of the different application of the specific stipulations of this power law forms, we refer to Hristov [34].

In the following we restrict the polynomial expansion of the conductivity to $i_{\max }=1$, so that terms of an order higher than explicitly shown on the right side of (3) will be neglected.

The heat equation which describes the nonlinear heat propagation is taken the form

$$
\sum_{k=1}^{3} \frac{\partial}{\partial x_{k}}\left(\kappa \frac{\partial T}{\partial x_{k}}\right)=\rho_{p} \frac{\partial T}{\partial t}
$$

where $\rho_{p}=\rho_{p}(\mathbf{x})=c_{p} \rho$ is the product of the specific heat capacity $c_{p}=c_{p}(\mathbf{x})$ and the mass density $\rho=\rho(\mathbf{x})$ of the material. While $\rho_{p}$ is taken to be independent from the temperature in this article, this shall be noted that this parameter reveals a strong temperature-dependence in the case of phase-changes [25]. After the substitution the specific stipulation of the thermal conductivity (3) for $i_{\max }=1$ into the heat equation (4), we obtain a nonlinear heat equation in the form

$$
\sum_{k=1}^{3}\left\{a_{0} \frac{\partial^{2} T}{\partial x_{k}^{2}}+a_{1}\left[\left(\frac{\partial T}{\partial x_{k}}\right)^{2}+T \frac{\partial^{2} T}{\partial x_{k}^{2}}\right]\right\}=\rho_{p} \frac{\partial T}{\partial t} .
$$

This form of the heat equation in a homogeneous and isotropic solid will serve as a basis for our considerations in the now following section, in which we formulate the governing relations in the considered composite.

\subsection{Heat diffusion in a composite}

Let us consider the heat flux piece-wise for every constituent $\Omega^{(i)}$, where $i=1,2$. From (1), (3), and (5) we obtain the following set of equations,

$$
\begin{gathered}
q^{(i)}=-\kappa^{(i)} \frac{\partial T^{(i)}}{\partial x_{k}}, \quad k=1,2,3 \\
\kappa^{(i)}\left(T^{(i)}(\mathbf{x}, t)\right)=a_{0}^{(i)}+a_{1}^{(i)} T^{(i)}(\mathbf{x}, t), \\
\sum_{k=1}^{3}\left\{a_{0}^{(i)} \frac{\partial^{2} T^{(i)}}{\partial x_{k}^{2}}+a_{1}^{(i)}\left[\left(\frac{\partial T^{(i)}}{\partial x_{k}}\right)^{2}+T^{(i)} \frac{\partial^{2} T^{(i)}}{\partial x_{k}^{2}}\right]\right\}=\rho_{p}^{(i)} \frac{\partial T^{(i)}}{\partial t}
\end{gathered}
$$

where $T^{(i)}=T^{(i)}(\mathbf{x}, t)$ is the temperature, $\rho_{p}^{(i)}$ is the product of the specific heat capacity and the mass density, $q^{(i)}=q^{(i)}(T(\mathbf{x}, t))$ is the heat flux, and $\kappa^{(i)}=\kappa^{(i)}\left(T^{(i)}(\mathbf{x}, t)\right)$ in the constituent $\Omega^{(i)}$. In (6b) and (6c), the parameters $a_{0}^{(i)}$ are the temperature-independent term of the thermal conductivity, and the parameters $a_{1}^{(i)}$ define the change of the conductivity $\kappa^{(i)}$ with the temperature.

Let us assume thermal resistance at the common interface $\partial \Omega^{(1,2)}$ of the constituents $\Omega^{(1)}$ and $\Omega^{(2)}$. Such resistance might for example results from imperfect bonding between the components, 
cracks in the interface, corrosion, or from a thin interphase material such as a coating. The interface conditions are taken into account by two conjugate conditions, which are now specified in detail.

- Equality of the heat flux at $\partial \Omega^{(1,2)}$ : The heat flux in both constituents is taken to be equal at their common interface $\partial \Omega^{(1,2)}$, so that together with (6a) we can formulate this conjugate condition as follows:

$$
\left.\left\{\kappa^{(1)} \frac{\partial T^{(1)}}{\partial n_{\mathbf{x}}}=\kappa^{(2)} \frac{\partial T^{(2)}}{\partial n_{\mathbf{x}}}\right\}\right|_{\partial \Omega^{(1,2)}},
$$

where

$$
\frac{\partial}{\partial n_{\mathbf{x}}}=n_{1} \frac{\partial}{\partial x_{1}}+n_{2} \frac{\partial}{\partial x_{2}}+n_{3} \frac{\partial}{\partial x_{3}}
$$

is a directional derivative, and $n_{k}, k=1,2,3$, are the elements of the normal unit vector $\mathbf{n}$ to the interface $\partial \Omega^{(1,2)}$ (see Fig. 1). After substitution of the specific stipulation for the thermal conductivity $(6 \mathrm{~b})$ into (7), this equation takes the form

$$
\left.\left\{\left(a_{0}^{(1)}+a_{1}^{(1)} T^{(1)}\right) \frac{\partial T^{(1)}}{\partial n_{\mathbf{x}}}=\left(a_{0}^{(2)}+a_{1}^{(2)} T^{(2)}\right) \frac{\partial T^{(2)}}{\partial n_{\mathbf{x}}}\right\}\right|_{\partial \Omega^{(1,2)}} .
$$

- Temperature difference at $\partial \Omega^{(1,2)}$ : Different articles such as Quang et al. [26, 27] consider the differences in the temperature at $\partial \Omega^{(1,2)}$ as a linear function of the heat flux. In the present article we assume, that the temperature difference $\Delta T=T^{(2)}-T^{(1)}$ is related in a nonlinear way to the heat flux, and we apply the following model

$$
\left.\left\{ \pm \Delta T=\sum_{j=1}^{j_{\max }} b_{j}\left(\kappa^{(1)} \frac{\partial T^{(1)}}{\partial n_{\mathbf{x}}}\right)^{j}\right\}\right|_{\partial \Omega^{(1,2)}},
$$

where $b_{j}$ are constants which specify the quality of the interfacial resistance. If all constants $b_{j}=0$, then there is not thermal resistance at $\partial \Omega^{(1,2)}$. If the terms $b_{j}$ for $j \geq 2$ are neglected, then (10) reduces to the classical Kapitza model [23] for thermal resistance. On the left side of Eq. (10), the upper part of " \pm " belongs to the interface $\partial \Omega^{(1,2)}$, which is located in the positive $\mathbf{E}_{1}$-direction from the inclusion $\Omega^{(1)}$, and the bottom part " \pm " belongs to the interface $\partial \Omega^{(1,2)}$, which is located in the negative $\mathbf{E}_{1}$-direction from the inclusion $\Omega^{(1)}$.

In the following parts of this article, we consider the case of $j_{\max }=2$ in (10). After substitution of the proposed stipulation for the conductivity (6b) into (10), this equation takes the form

$$
\left.\left\{ \pm \Delta T=b_{1}\left[\left(a_{0}^{(1)}+a_{1}^{(1)} T^{(1)}\right) \frac{\partial T^{(1)}}{\partial n_{\mathbf{x}}}\right]+b_{2}\left[\left(a_{0}^{(1)}+a_{1}^{(1)} T^{(1)}\right) \frac{\partial T^{(1)}}{\partial n_{\mathbf{x}}}\right]^{2}\right\}\right|_{\partial \Omega^{(1,2)}}
$$

Let us rewrite the right-hand side of (11) as follows

$$
\begin{aligned}
& a_{0}^{(1)} b_{1} \frac{\partial T^{(1)}}{\partial n_{\mathbf{x}}}+a_{1}^{(1)} b_{1} T^{(1)} \frac{\partial T^{(1)}}{\partial n_{\mathbf{x}}}+\left[a_{0}^{(1)}\right]^{2} b_{2}\left(\frac{\partial T^{(1)}}{\partial n_{\mathbf{x}}}\right)^{2} \\
& +2 a_{0}^{(1)} a_{1}^{(1)} b_{2} T^{(1)}\left(\frac{\partial T^{(1)}}{\partial n_{\mathbf{x}}}\right)_{5}^{2}+\left[a_{1}^{(1)}\right]^{2} b_{2}\left[T^{(1)}\right]^{2}\left(\frac{\partial T^{(1)}}{\partial n_{\mathbf{x}}}\right)^{2} .
\end{aligned}
$$


If we assume a weak nonlinearity with $a_{0}^{(1)} \gg a_{1}^{(1)} T^{(1)}$ and $\left[a_{0}^{(1)}\right]^{2} \gg 2 a_{0}^{(1)} a_{1}^{(1)} T^{(1)}$, then we can take the last two terms in (12) to be negligibly small in comparison to the other terms. Applying this assumption, Eq. (11) becomes

$$
\left.\left\{ \pm \Delta T=a_{0}^{(1)} b_{1} \frac{\partial T^{(1)}}{\partial n_{\mathbf{x}}}+a_{1}^{(1)} b_{1} T^{(1)} \frac{\partial T^{(1)}}{\partial n_{\mathbf{x}}}+\left[a_{0}^{(1)}\right]^{2} b_{2}\left(\frac{\partial T^{(1)}}{\partial n_{\mathbf{x}}}\right)^{2}\right\}\right|_{\partial \Omega^{(1,2)}} .
$$

This thermal resistance model has some analogies to the nonlinear spring-layer bonding model for mechanical problems, which has been initially proposed by Goland \& Reissner [35]. This model is based on the assumption that the interfacial stress is a function of the gap in the displacements. Linear and non-linear versions of this model have been discussed, for example, in [36].

\section{Application of the asymptotic homogenization method to obtain the effective heat equa- tion}

In order to determine the effective or homogenized thermal properties of the composite, the boundary value problem which consists of the heat Eq. (6c) and conjugate conditions to describe the thermal resistance at the interface in Eqs. (9) and (13) is analyzed by the application of the AHM.

The size of the heterogeneities $\ell$ is considered to be much smaller than the macroscopic size $L$ of the considered problem, $\ell \ll L$. The ratio of the length $\ell$ to the length $L$ is defined by a small parameter $\epsilon$,

$$
L=\epsilon^{-1} \ell \text {. }
$$

Two types of Cartesian coordinate variables $\boldsymbol{\eta}$ and $\zeta$ are now introduced in the form

$$
\begin{aligned}
& \boldsymbol{\eta}=\mathbf{E}_{1} \eta_{1}+\mathbf{E}_{2} \eta_{2}+\mathbf{E}_{3} \eta_{3}, \\
& \boldsymbol{\zeta}=\mathbf{E}_{1} \zeta_{1}+\mathbf{E}_{2} \zeta_{2}+\mathbf{E}_{3} \zeta_{3} .
\end{aligned}
$$

The coordinate variables $\boldsymbol{\eta}$ are denoted as the slow coordinate variables, and they measures the temperature in the area of interest, while the coordinate variables $\zeta$ are denoted as the fast coordinates, and they measure the temperature in the considered unit cell. These fast and slow coordinate variables are related to the original coordinate variables $\mathbf{x}$ via (see, i.e., Bensoussan et al. [9])

$$
\mathbf{x} \rightarrow \boldsymbol{\eta}, \quad \zeta=\epsilon^{-1} \boldsymbol{\eta} .
$$

The boundary value problem contains first and second derivatives with respect to the elements of $\mathbf{x}$, and directional derivatives normal to the interface. In the notations of the slow and fast coordinates, these derivatives are also substitutes as follows,

$$
\begin{aligned}
\frac{\partial}{\partial x_{k}} \rightarrow \frac{\partial}{\partial \eta_{k}}+\frac{1}{\epsilon} \frac{\partial}{\partial \zeta_{k}}, & k=1,2,3, \\
\frac{\partial^{2}}{\partial x_{k}^{2}} \rightarrow \frac{\partial^{2}}{\partial \eta_{k}^{2}}+\frac{2}{\epsilon} \frac{\partial^{2}}{\partial \eta_{k} \partial \zeta_{k}}+\frac{1}{\epsilon^{2}} \frac{\partial^{2}}{\partial \zeta_{k}^{2}}, & k=1,2,3, \\
\frac{\partial}{\partial n_{\mathbf{x}}} \rightarrow \frac{\partial}{\partial n_{\eta}}+\frac{1}{\epsilon} \frac{\partial}{\partial n_{\zeta}}, &
\end{aligned}
$$


where

$$
\begin{aligned}
& \frac{\partial}{\partial n_{\eta}}=n_{1} \frac{\partial}{\partial \eta_{1}}+n_{2} \frac{\partial}{\partial \eta_{2}}+n_{3} \frac{\partial}{\partial \eta_{3}}, \\
& \frac{\partial}{\partial n_{\zeta}}=n_{1} \frac{\partial}{\partial \zeta_{1}}+n_{2} \frac{\partial}{\partial \zeta_{2}}+n_{3} \frac{\partial}{\partial \zeta_{3}} .
\end{aligned}
$$

In terms of the slow coordinate variables $\boldsymbol{\eta}$, the lengths of the unit cell remains $\ell_{k}$ in the direction $\mathbf{E}_{k}$, where $k=1,2,3$. In the notation of the fast coordinate variables $\zeta$, the length of the unit cell then becomes $L_{k}$, which is related to the lengths $\ell_{k}$ by the small parameter in the following way:

$$
L_{k}=\epsilon^{-1} \ell_{k}, \quad k=1,2,3 .
$$

The distribution of the temperature $T^{(i)}$ in the constituent $\Omega^{(i)}$ is now searched in form of an asymptotic expansion in powers of the small parameter $\epsilon$,

$$
T^{(i)}=\sum_{n=0} \epsilon^{n} T_{n}^{(i)} .
$$

The first summand $T_{0}^{(i)}=T_{0}^{(i)}(\boldsymbol{\eta}, t)=T_{0}$ in (20) is the homogenized term, and it is a function of the slow coordinate $\eta$, which replaces the original coordinate $\mathbf{x}$, and time $t$ (see Bakhvalov\& Panasenko [37]). The then following terms $T_{n}^{(i)}=T_{n}^{(i)}(\boldsymbol{\eta}, \boldsymbol{\zeta}, t)$ for $n=1,2, \ldots$ are correction terms of the order $\epsilon^{n}$, which therefore depend on both the slow coordinate variables $\eta$ and time $t$ as the homogenized leading term as well as on the fast coordinate variables $\zeta$. The periodicity of the composite structure results into the following periodicity condition for the corrections terms of the temperature field in terms of the fast coordinate variables $\zeta$,

$$
T_{n}^{(i)}(\boldsymbol{\eta}, \boldsymbol{\zeta}, t)=T_{n}^{(i)}(\boldsymbol{\eta}, \boldsymbol{\zeta}+\mathbf{L}, t), \quad n=1,2, \ldots
$$

where $\mathbf{L}$ is a translation vector in the form

$$
\mathbf{L}=\mathbf{E}_{1} \lambda_{1} L_{1}+\mathbf{E}_{2} \lambda_{2} L_{2}+\mathbf{E}_{3} \lambda_{3} L_{3},
$$

where $\lambda_{k}= \pm 1, \pm 2, \ldots$ are integers, $k=1,2,3$. The unit cell with the length $L_{k}=\epsilon^{-1} \ell_{k}$ in terms of the fast coordinate variables $\zeta_{k}$ is symmetric with respect to the axes of the coordinate system $\left\{\mathbf{E}_{1}, \mathbf{E}_{2}, \mathbf{E}_{3}\right\}$.

\subsection{Homogenized heat equation of different asymptotic orders}

In the present section we apply the AHM to the boundary value problem in Sec. 2.2. After substitution of the asymptotic expansion of the temperature field (20) into the heat Eq. (6c) and applying the derivatives in (17), we derive a heat equation in terms of the slow and fast coordinate 
variables in the form

$$
\begin{aligned}
& \sum_{k=1}^{3}\left\{a_{0}^{(i)} \sum_{n} \epsilon^{n}\left(\frac{\partial^{2} T_{n}^{(i)}}{\partial \eta_{k}^{2}}+\frac{2}{\epsilon} \frac{\partial^{2} T_{n}^{(i)}}{\partial \eta_{k} \partial \zeta_{k}}+\frac{1}{\epsilon^{2}} \frac{\partial^{2} T_{n}^{(i)}}{\partial \zeta_{k}^{2}}\right)\right. \\
& +a_{1}^{(i)}\left[\sum_{n} \epsilon^{n}\left(\frac{\partial T_{n}^{(i)}}{\partial \eta_{k}}+\frac{1}{\epsilon} \frac{\partial T_{n}^{(i)}}{\partial \zeta_{k}}\right)\right]^{2} \\
& \left.+a_{1}^{(i)}\left(\sum_{n} \epsilon^{n} T_{n}^{(i)}\right)\left[\sum_{n} \epsilon^{n}\left(\frac{\partial^{2} T_{n}^{(i)}}{\partial \eta_{k}^{2}}+\frac{2}{\epsilon} \frac{\partial^{2} T_{n}^{(i)}}{\partial \eta_{k} \partial \zeta_{k}}+\frac{1}{\epsilon^{2}} \frac{\partial^{2} T_{n}^{(i)}}{\partial \zeta_{k}^{2}}\right)\right]\right\} \\
& =\rho_{p}^{(i)} \sum_{n} \epsilon^{n} \frac{\partial T_{n}^{(i)}}{\partial t}
\end{aligned}
$$

The thermal resistance of the interface $\partial \Omega^{(1,2)}$ is modeled by the conjugate conditions in (9) and (13), and we also want to present these conditions in terms of the slow and fast coordinate variables.

- Equality of the heat flux at $\partial \Omega^{(1,2)}$ : In terms of slow and fast coordinates, the conjugate condition Eq. (9) takes the form,

$$
\begin{aligned}
& \left\{a_{0}^{(1)}\left[\sum_{n} \epsilon^{n}\left(\frac{\partial T_{n}^{(1)}}{\partial n_{\eta}}+\frac{1}{\epsilon} \frac{\partial T_{n}^{(1)}}{\partial n_{\zeta}}\right)\right]\right. \\
& +a_{1}^{(1)}\left(\sum_{n} \epsilon^{n} T_{n}^{(1)}\right)\left[\sum_{n} \epsilon^{n}\left(\frac{\partial T_{n}^{(1)}}{\partial n_{\eta}}+\frac{1}{\epsilon} \frac{\partial T_{n}^{(1)}}{\partial n_{\zeta}}\right)\right] \\
& =a_{0}^{(2)}\left[\sum_{n} \epsilon^{n}\left(\frac{\partial T_{n}^{(2)}}{\partial n_{\eta}}+\frac{1}{\epsilon} \frac{\partial T_{n}^{(2)}}{\partial n_{\zeta}}\right)\right] \\
& +\left.a_{1}^{(2)}\left(\sum_{n} \epsilon^{n} T_{n}^{(2)}\right)\left[\sum_{n} \epsilon^{n}\left(\frac{\partial T_{n}^{(2)}}{\partial n_{\eta}}+\frac{1}{\epsilon} \frac{\partial T_{n}^{(2)}}{\partial n_{\zeta}}\right)\right\}\right|_{\partial \Omega^{(1,2)}}
\end{aligned}
$$

- Temperature difference at $\partial \Omega^{(1,2)}$ : Equation (13) can be rewritten as

$$
\begin{aligned}
\left\{ \pm \sum_{n} \epsilon^{n} \Delta T_{n}\right. & =a_{0}^{(1)} b_{1}\left[\sum_{n} \epsilon^{n}\left(\frac{\partial T_{n}^{(1)}}{\partial n_{\eta}}+\frac{1}{\epsilon} \frac{\partial T_{n}^{(1)}}{\partial n_{\zeta}}\right)\right] \\
& +a_{1}^{(1)} b_{1}\left(\sum_{n} \epsilon^{n} T_{n}^{(1)}\right)\left[\sum_{n} \epsilon^{n}\left(\frac{\partial T_{n}^{(1)}}{\partial n_{\eta}}+\frac{1}{\epsilon} \frac{\partial T_{n}^{(1)}}{\partial n_{\zeta}}\right)\right] \\
& \left.+\left[a_{0}^{(1)}\right]^{2} b_{2}\left[\sum_{n} \epsilon^{n}\left(\frac{\partial T_{n}^{(1)}}{\partial n_{\eta}}+\frac{1}{\epsilon} \frac{\partial T_{n}^{(1)}}{\partial n_{\zeta}}\right)\right]^{2}\right\}\left.\right|_{\partial \Omega^{(1,2)}},
\end{aligned}
$$

where $\Delta T_{n}=T_{n}^{(2)}-T_{n}^{(1)}$ is the temperature difference of the $n$-th order terms of expansion of the temperature field at the interface $\partial \Omega^{(1,2)}$.

In the equations (23) - (25) we find terms of different orders $\epsilon^{n}$ which result from the expansion of the temperature field in (20), and which might result from the differences of the material parameter values. For example, if the ratio of the thermal conductivities $\kappa^{(2)} / \kappa^{(1)}$ of the constituents 
is of the $n$-th order of the small parameters $\epsilon$, then this has to be taken into account in deriving heat equations of the different asymptotic orders. Cherednichenko et al. [38] and Gałka et al. [16] discuss material parameters of different asymptotic orders, which gives a splitting scheme that may result into non-local effects in the effective equations. In the boundary value problem (23) - (25), the material parameters of the different constituents and the parameters in the bonding conditions are taken to be of the same asymptotic order

$$
a_{0}^{(1)} \sim a_{0}^{(2)}, \quad a_{1}^{(1)} \sim a_{1}^{(2)} .
$$

In order to derive the effective thermal properties of different asymptotic orders, we subdivide the boundary value problem in (23) - (25) into a recurrent system of equations of different orders with respect to the small parameter $\epsilon$. Such splitting of the heat Eq. (23) gives

$$
\begin{aligned}
& \sum_{k=1}^{3}\left\{a_{0}^{(i)}\left(\frac{\partial^{2} T_{n-2}^{(i)}}{\partial \eta_{k}^{2}}+2 \frac{\partial^{2} T_{n-1}^{(i)}}{\partial \eta_{k} \partial \zeta_{k}}+\frac{\partial^{2} T_{n}^{(i)}}{\partial \zeta_{k}^{2}}\right)\right. \\
& +a_{1}^{(i)}\left[\sum_{m=0}^{n-2}\left(\frac{\partial T_{m}^{(i)}}{\partial \eta_{k}}+\frac{\partial T_{m+1}^{(i)}}{\partial \zeta_{k}}\right)\left(\frac{\partial T_{n-m-2}^{(i)}}{\partial \eta_{k}}+\frac{\partial T_{n-m-1}^{(i)}}{\partial \zeta_{k}}\right)\right] \\
& \left.+a_{1}^{(i)}\left[\sum_{m=0}^{n-2} T_{m}^{(i)}\left(\frac{\partial^{2} T_{n-m-2}^{(i)}}{\partial \eta_{k}^{2}}+2 \frac{\partial^{2} T_{n-m-1}^{(i)}}{\partial \eta_{k} \partial \zeta_{k}}+\frac{\partial^{2} T_{n-m}^{(i)}}{\partial \zeta_{k}^{2}}\right)\right]\right\}=\rho_{p}^{(i)} \frac{\partial T_{n-2}^{(i)}}{\partial t},
\end{aligned}
$$

where $n=1,2, \ldots$ and $T_{n-2}^{(i)}=T_{0}$. In the case of a negative subscript in one of the temperature terms, this terms will become equal to zero, e.g., $T_{-1}^{(i)}=0$.

Let us apply the separation of the terms of different orders the interface conjugate conditions.

- Equality of the heat flux at $\partial \Omega^{(1,2)}$ : If we apply this splitting scheme to (24), then we obtain

$$
\begin{aligned}
& \left\{a_{0}^{(1)}\left(\frac{\partial T_{n-1}^{(1)}}{\partial n_{\eta}}+\frac{\partial T_{n}^{(1)}}{\partial n_{\zeta}}\right)+a_{1}^{(1)}\left[\sum_{m=0}^{n-1} T_{m}^{(1)}\left(\frac{\partial T_{n-m-1}^{(1)}}{\partial n_{\eta}}+\frac{\partial T_{n-m}^{(1)}}{\partial n_{\zeta}}\right)\right]\right. \\
& \left.=a_{0}^{(2)}\left(\frac{\partial T_{n-1}^{(2)}}{\partial n_{\eta}}+\frac{\partial T_{n}^{(2)}}{\partial n_{\zeta}}\right)+a_{1}^{(2)}\left[\sum_{m=0}^{n-1} T_{m}^{(2)}\left(\frac{\partial T_{n-m-1}^{(2)}}{\partial n_{\eta}}+\frac{\partial T_{n-m}^{(2)}}{\partial n_{\zeta}}\right)\right]\right\}\left.\right|_{\partial \Omega^{(1,2)}} .
\end{aligned}
$$

- Temperature difference at $\partial \Omega^{(1,2)}$ : For (25) we obtain

$$
\begin{aligned}
& \left\{ \pm \epsilon\left(T_{n}^{(2)}-T_{n}^{(1)}\right)=a_{0}^{(1)} b_{1}\left(\frac{\partial T_{n-1}^{(1)}}{\partial n_{\eta}}+\frac{\partial T_{n}^{(1)}}{\partial n_{\zeta}}\right)\right. \\
& +a_{1}^{(1)} b_{1} \sum_{m=0}^{n-1} T_{m}^{(1)}\left(\frac{\partial T_{n-m-1}^{(1)}}{\partial n_{\eta}}+\frac{\partial T_{n-m}^{(1)}}{\partial n_{\zeta}}\right) \\
& \left.+\left[a_{0}^{(1)}\right]^{2} b_{2}\left[\sum_{m=0}^{n-2}\left(\frac{\partial T_{m}^{(i)}}{\partial n_{\eta}}+\frac{\partial T_{m+1}^{(i)}}{\partial n_{\zeta}}\right)\left(\frac{\partial T_{n-m-2}^{(i)}}{\partial n_{\eta}}+\frac{\partial T_{n-m-1}^{(i)}}{\partial n_{\zeta}}\right)\right]\right\}\left.\right|_{\partial \Omega^{(1,2)}} .
\end{aligned}
$$

On the left-hand side of (29), we find the small parameter $\epsilon$ as a factor. To derive the different-order terms in the expansion of the temperature field in (20), we start with the lowest reasonable order $n=1$. If we would apply $\pm\left(T_{n-1}^{(2)}-T_{n-1}^{(1)}\right)$ instead of $\pm \epsilon\left(T_{n}^{(2)}-T_{n}^{(1)}\right)$, then the left side in Eq. (29) would be equal to zero for $n=1$, because $T_{0}^{(2)}=T_{0}^{(1)}=T_{0}$. 
We have to choose ansatzes for $T_{n}^{(i)}$, which satisfy the conditions (27) - (29). Once these ansatzes are found and their integration constants have been determined by the conjugate conditions, the homogenizing operator

$$
\langle\cdot\rangle=\frac{1}{V} \int_{-\frac{L_{3}}{2}}^{\frac{L_{3}}{2}} \int_{-\frac{L_{2}}{2}}^{\frac{L_{2}}{2}} \int_{-\frac{L_{1}}{2}}^{\frac{L_{1}}{2}}(\cdot) \mathrm{d} \zeta_{1} \mathrm{~d} \zeta_{2} \mathrm{~d} \zeta_{3},
$$

is applied to both sides of (27) in order to determine the homogenized or effective material parameters, where $V=L_{1} L_{2} L_{3}$.

In the following, we simplify the problem under consideration, and we take the unit cell is cubic with the side lengths $\ell_{1}=\ell_{2}=\ell_{3}=\ell$ in the notation of the slow coordinate variables, and $L_{1}=L_{2}=L_{3}=L$ in the notation of the fast coordinate variables.

We have presented a method based on asymptotic homogenization to derive the effective heat equations of different asymptotic orders, which also take nonlinear effects into account. The degree of nonlinearity results from two sources, the amount of terms, which are taken into account in the thermal conductivity model (1), and the number of terms which are taken into account in the thermal resistance model for the interface $\partial \Omega^{(1,2)}$ in $(11)$. The here proposed asymptotic homogenization paradigm allows to study a wide range of thermal effects. In the following sections we apply this method to study composites with a large volume fractions of the inclusions close to the maximum volume fraction, and small volume fractions of the inclusions.

- In Sec. 4 we apply the close packing model, which is also denoted as the lubrication theory to study the case of large inclusions. In this section, we mainly study the case of large spherical inclusions. We will show, that the obtained results applicable to problems of heat diffusion in arbitrary directions in the $\mathbf{E}_{1}-\mathbf{E}_{2}$-plane for both spherical inclusions and cylindrical inclusions. At the end of that section, we also briefly discuss layered composite materials.

- In Sec. 5 we apply the three-phase model in order to study the effective properties of the composite when the volume fractions of the inclusions are considered to be small. Two different types of inclusions will be considered. In the first part we study heat transfer in directions perpendicular to the parallel cylindrical inclusions. In the second case, we apply the three-phase model in order to obtain the effective thermal properties of composites with spherical inclusions.

The herein studied limiting cases of large and small volume fractions of the inclusions might be matched in order to obtain the effective thermal properties of the material for intermediate volume fractions for the inclusion, for example by the application of two-point Padé approximants [39]. This matching the limiting solutions lies beyond the scope of the present article.

\section{4. Densely packed composites: using of lubrication theory}

The main idea of the lubrication theory relies on changing the boundary value problem with possibly uneven surfaces of the inclusion from the original space into the space of a simplified geometry. Figure 2 shows two examples for inclusions with uneven geometries. It should be noted that while employing the inclusions with a large size, these inclusions are almost in touch and the most important physical processes (for instance, heat flux) take place in a very thin domain. The 

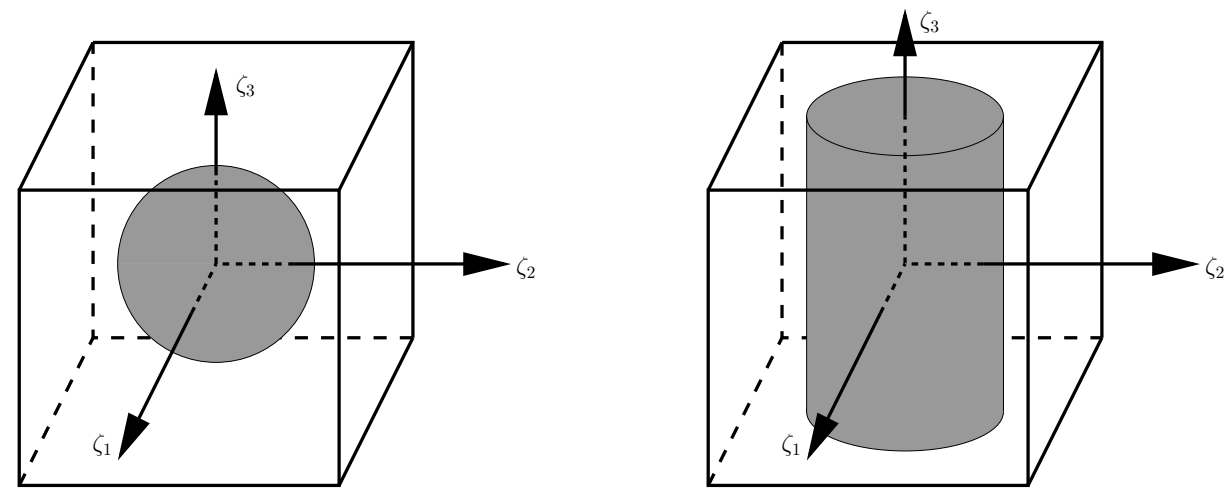

Figure 2: Two cubic unit cells in terms of the fast coordinate variables $\zeta_{k}$, where $k=1,2,3$. The left unit cell has a spherical inclusion, and the right unit cell a cylindrical inclusion with an axis oriented in the $\zeta_{3}$-direction.

lubrication theory plays a key role in calculations of the high contrast, densely packed composites. In order to define the character of an asymptotic behavior, one needs to employ methods, which clearly exhibit the physical behavior of the processes, which occur in the investigated composites. For the high contrast, densely packed composites, the most adequate approaches are represented by network approximation methods [40] and lubrication theory [21]. Note that the lubrication theory is more suitable in our case, because it allows to obtain solutions for a large interval of parameters, for instance, for a finite but large heat conductivity of the inclusions. Such model has been justified and applied in different studies on asymptotic homogenization, for example by Kalamkarov et al. [22] for heat diffusion problems, for by Andrianov et al. [11, 41] for wave propagation problems. Frankel \& Acrivos [42] applied this model to determine the effective viscosity of a concentrated suspension of solid spheres. This approach allows us to consider the heat problem direction-wise in the $\mathbf{E}_{1}, \mathbf{E}_{2}$, and $\mathbf{E}_{3}$ directions. We use terms lubrication theory or densely packed model approach, which is also dented as the concentrated suspension model or the lubrication approximation by Christensen [21]. From the mathematical standpoint we apply the thin layer approach (see Tayler [43]). This approach results into simplified models by assuming that the length scales in a direction $\mathbf{E}_{k}$ are much smaller than in the directions normal to it. The used formal procedure is to rescale the $x_{k}$ variable with a small parameter $\epsilon$ expressing the ration of the relative length scales. For a cubic unit cell with a large inclusion in its center, the symmetry of the presented problem in the space of a simplified geometry allows us to consider a single direction $\mathbf{E}_{k}$, where $k=1,2,3$, and to generalize the results for the three-dimensional case. Figure 3 shows a brief example of the lubrication theory model for heat propagation in the $\zeta_{1}$-direction. The figure shows a cross-sectional area of the cubic unit cell in the $\mathbf{E}_{1}-\mathbf{E}_{2}$ plane (all considerations for the $\mathbf{E}_{1}-\mathbf{E}_{3}$ plane are analogue to these considerations). The original inclusion geometry is replaced by an inclusion strip of the length $L^{(1)}$. In the matrix strips $\Omega_{\perp}^{(2)}$, which are perpendicular to the direction of heat diffusion, the changes in the temperature field $T_{n}^{(2)}$ in $\zeta_{1}$-directions are dominant in comparison to the changes in $\zeta_{2}$-direction, and in the matrix strips $\Omega_{\|}^{(2)}$, which are parallel to the direction of heat diffusion, the changes in the temperature field in $\zeta_{2}$-directions are dominant in comparison to the changes in $\zeta_{1}$-direction (see, for example, 


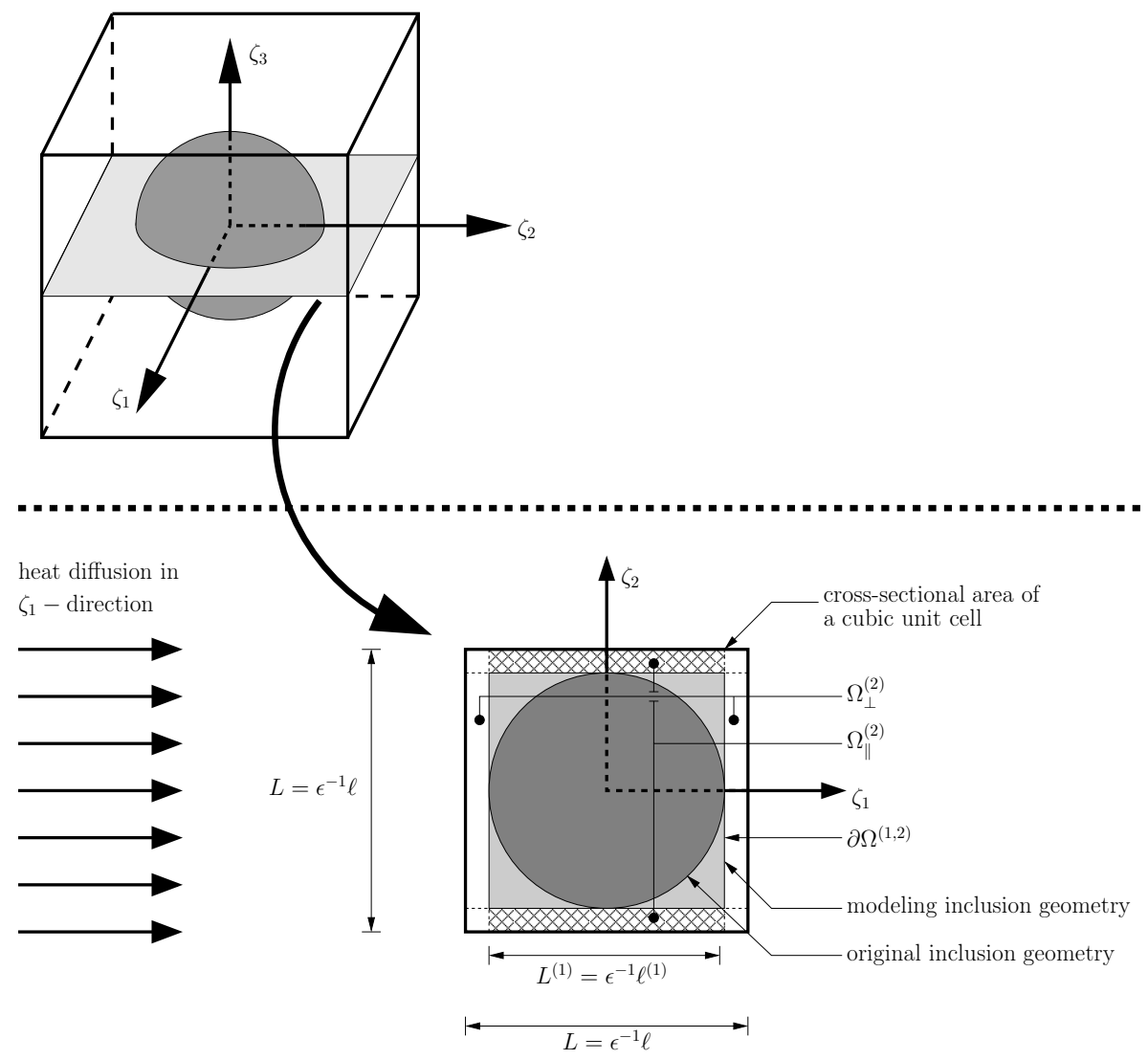

Figure 3: Lubrication theory model for large volume fractions of the inclusion $\Omega^{(1)}$ and heat propagation into the $\zeta_{1-}$ direction: The figure shows a cross-sectional area of the cubic unit cell in the $\mathbf{E}_{1}-\mathbf{E}_{2}$ plane (all considerations for the $\mathbf{E}_{1}-\mathbf{E}_{3}$ plane are analogue to these considerations). The original inclusion geometry is replaced by an inclusion strip of the length $L^{(1)}$, which for a spherical inclusion corresponds to the diameter of the spherical inclusion. In the matrix strips $\Omega_{\perp}^{(2)}$ the changes in the temperature field $T_{n}^{(2)}$ in $\zeta_{1}$-directions are dominant in comparison to the changes in $\zeta_{2}$-direction, and in the matrix strips $\Omega_{\|}^{(2)}$ the changes in the temperature field in $\zeta_{2}$-directions are dominant in comparison to the changes in $\zeta_{1}$-direction.

Andrianov et al. [44]):

$$
\begin{array}{ll}
\text { matrix strip } \Omega_{\perp}^{(2)} & \frac{\partial^{2} T_{n}^{(2)}}{\partial \zeta_{1}^{2}}>>\frac{\partial^{2} T_{n}^{(2)}}{\partial \zeta_{2}^{2}} \\
\text { matrix strip } \Omega_{\|}^{(2)} & \frac{\partial^{2} T_{n}^{(2)}}{\partial \zeta_{2}^{2}}>>\frac{\partial^{2} T_{n}^{(2)}}{\partial \zeta_{1}^{2}}
\end{array}
$$

235 Analogously for the considerations in the $\mathbf{E}_{1}-\mathbf{E}_{3}$ plane, for the strip $\Omega_{\perp}^{(2)}$ we obtain $\frac{\partial^{2} T_{n}^{(2)}}{\partial \zeta_{1}^{2}}>>$ 236 $\frac{\partial^{2} T_{n}^{(2)}}{\partial \zeta_{3}^{2}}$, and for the strip $\Omega_{\|}^{(2)}$ we obtain $\frac{\partial^{2} T_{n}^{(2)}}{\partial \zeta_{3}^{2}}>>\frac{\partial^{2} T_{n}^{(2)}}{\partial \zeta_{1}^{2}}$. For heat transfer in the directions of $\zeta_{2}$ ${ }_{237}$ and $\zeta_{3}$, these considerations are applied in a similar fashion. A justification of the lubrication 
theory model for spherical inclusions and cylindrical inclusions can be found, for example, in Christensen \& Lo [19].

Let us substitute $n=1$ into the heat equation (27), and we obtain for the constituent $\Omega^{(i)}$

$$
\frac{\partial^{2} T_{1}^{(i)}}{\partial \zeta_{1}^{2}}+\frac{\partial^{2} T_{1}^{(i)}}{\partial \zeta_{2}^{2}}+\frac{\partial^{2} T_{1}^{(i)}}{\partial \zeta_{3}^{2}}=0, \quad i=1,2 .
$$

Bakhvalov \& Panasenko [37] have shown that for such symmetric unit cell the condition (21) can be replaced by two boundary conditions in the center and at the outer boundaries of the unit cell in the form

$$
\begin{aligned}
& \left.\left\{T_{n}^{(1)}=0\right\}\right|_{\zeta_{k}=0}, \\
& \left.\left\{T_{n}^{(1)}=0\right\}\right|_{\zeta_{k}= \pm \frac{L_{k}}{2}} .
\end{aligned}
$$

This replacement has been applied in different works on the asymptotic analysis of the effective properties of periodic materials, for example, in [11] for the effective mechanical properties of composites, and in [29] for the effective thermal properties.

By applying the lubrication theory model to a cubic unit cell with a large inclusion (see Fig. 3 and Eqs. (31)) for the separate considerations in the $\mathbf{E}_{k}$ directions, where $k=1,2,3$, we can replace the ansatz in (32) by the following set of ansatzes for $T_{1}^{(i)}$,

$$
\frac{\partial^{2} T_{1}^{(i)}}{\partial \zeta_{k}^{2}}=0 \quad \Leftrightarrow \quad T_{1}^{(i)}=\left(c_{k, 1}^{(i)}+c_{k, 2}^{(i)} \zeta_{k}\right) \frac{\partial T_{0}}{\partial \eta_{k}}, \quad k=1,2,3, \quad i=1,2,
$$

where $c_{k, 1}^{(i)}$ and $c_{k, 2}^{(i)}$ are four integration constants for each direction of the heat flux $\mathbf{E}_{k}$.

In the interface conjugate conditions (28) and (29) we find the directional derivatives $\frac{\partial}{\partial n_{\eta}}$ and $\frac{\partial}{\partial n_{\zeta}}$. For the asymptotic lubrication model these derivatives take the forms $\frac{\partial}{\partial n_{\eta}}=\frac{\partial}{\partial \eta_{k}}$ and $\frac{\partial}{\partial n_{\zeta}}=$ $\frac{\partial}{\partial \zeta_{k}}$, where $k=1,2,3$. The conjugate conditions (28) and (29) in the interface $\partial \Omega^{(1,2)}$ then take the following forms:

- Equality of the heat flux at $\partial \Omega^{(1,2)}$ : For $n=1$, conjugate condition (28) takes the following form after collecting the terms and canceling out $\frac{\partial T_{0}}{\partial \eta_{k}}$ on both sides:

$$
\left[a_{0}^{(1)}+a_{1}^{(1)} T_{0}\right]\left(1+c_{k, 2}^{(1)}\right)=\left[a_{0}^{(2)}+a_{1}^{(2)} T_{0}\right]\left(1+c_{k, 2}^{(2)}\right), \quad k=1,2,3 .
$$

- Temperature fifference at $\partial \Omega^{(1,2)}:$ For $n=1$, the conjugate condition (29) becomes

$$
\epsilon\left[\left(c_{1, k}^{(2)}-c_{1, k}^{(1)}\right) \pm \frac{L_{1}}{2}\left(c_{2, k}^{(2)}-c_{2, k}^{(1)}\right)\right]=b_{1}\left[a_{0}^{(1)}+a_{1}^{(1)} T_{0}\right]\left(1+c_{2, k}^{(1)}\right), \quad k=1,2,3,
$$

where $L_{1}=\epsilon^{-1} \ell_{1}$. Note that in the here considered first approximation equation (36) is independent from $b_{2}$. The upper sign in " \pm " results from the boundary condition at $\zeta_{k}=$ $L_{1} / 2$, and the bottom sign results from the boundary condition at $\zeta_{k}=-L_{1} / 2$.

In the ansatz for $T_{1}^{(i)}$ in Eq. (34) we find four constants $c_{k, 1}^{(i)}$ and $c_{k, 2}^{(i)}$, which are determined from the four conjugate conditions (33), (35) and (36). 


$$
\begin{aligned}
& \frac{1}{L^{3}} \sum_{k=1}^{3} \int_{-\frac{L}{2}}^{\frac{L}{2}} \int_{-\frac{L}{2}}^{\frac{L}{2}} \int_{-\frac{L}{2}}^{\frac{L}{2}} a_{0}^{(i)}\left(\frac{\partial^{2} T_{0}}{\partial \eta_{k}^{2}}+\frac{\partial^{2} T_{1}^{(i)}}{\partial \eta_{k} \partial \zeta_{k}}\right)+a_{1}^{(i)}\left(\frac{\partial T_{0}}{\partial \eta_{k}}+\frac{\partial T_{1}^{(i)}}{\partial \zeta_{k}}\right)^{2} \\
+ & a_{1}^{(i)} T_{0}\left(\frac{\partial^{2} T_{0}}{\partial \eta_{k}^{2}}+\frac{\partial^{2} T_{1}^{(i)}}{\partial \eta_{k} \partial \zeta_{k}}\right) \mathrm{d} \zeta_{k} \mathrm{~d} \zeta_{r} \mathrm{~d} \zeta_{s} \\
= & \frac{1}{L^{3}} \sum_{k=1}^{3} \int_{-\frac{L}{2}}^{\frac{L}{2}} \int_{-\frac{L}{2}}^{\frac{L}{2}} \int_{-\frac{L}{2}}^{\frac{L}{2}} \rho_{p}^{(i)} \frac{\partial T_{0}}{\partial t} \mathrm{~d} \zeta_{k} \mathrm{~d} \zeta_{r} \mathrm{~d} \zeta_{s}, \quad r \neq k, r \neq s, s \neq k .
\end{aligned}
$$

Although the substitution $n=2$ has been applied, all terms which contain the correction terms $T_{2}^{(2)}$ have been canceled out (also see Appendix A and [29]).

We substitute the now known forms of $T_{1}^{(i)}$ into (37), and expand the result into a McLaurin series for the homogenized term $T_{0}$ of the temperature field. After neglecting all terms of a higher order than the terms in the given boundary value problem, we obtain a homogenized heat equation in the form of the homogenized parameters $\langle\cdot\rangle$ of the order $O\left(\epsilon^{0}\right)$,

$$
\sum_{k=1}^{3}\left\{\left\langle a_{0}\right\rangle \frac{\partial^{2} T_{0}}{\partial \eta_{k}^{2}}+\left\langle a_{1}\right\rangle\left[\left(\frac{\partial T_{0}}{\partial \eta_{k}}\right)^{2}+T_{0} \frac{\partial^{2} T_{0}}{\partial \eta_{k}^{2}}\right]+O\left(\epsilon^{1}\right)\right\}=\left\langle\rho_{p}\right\rangle \frac{\partial T_{0}}{\partial t},
$$

where on the left side $\left\langle a_{0}\right\rangle$ and $\left\langle a_{1}\right\rangle$ are homogenized parameters which result from thermal conductivities of the constituents, the geometry of the unit cell, and the bonding factors. On the right side, $\left\langle\rho_{p}\right\rangle$ is a homogenized parameter which results from the product of the specific heat capacities and the mass densities of the constituents and the geometry. These homogenized parameters have the forms

$$
\begin{aligned}
& \left\langle a_{0}\right\rangle=\frac{\left(\gamma_{1}+\alpha_{1}\right) \ell}{\left[N+N_{b}\right]^{2}}, \\
& \left\langle a_{1}\right\rangle=\frac{\beta_{1} \ell}{\left[N+N_{b}\right]^{2}}, \\
& \left\langle\rho_{p}\right\rangle=\frac{\rho_{p}^{(1)} \ell_{k}^{(1)}+\rho_{p}^{(2)} \ell_{k}^{(2)}}{\ell}, \quad k=1,2,3,
\end{aligned}
$$

where $\ell_{k}^{(1)}$ for $k=1,2,3$ are the lengths of the inclusions in the $\mathbf{E}_{k}$ directions in terms of the slow coordinate variables, $\ell_{k}^{(2)}=\ell_{k}-\ell_{k}^{(1)}$, and

$$
\begin{aligned}
& \alpha_{1}=\left[a_{0}^{(1)}\right]^{2} a_{0}^{(2)} \ell_{k}^{(2)}+a_{0}^{(1)}\left[a_{0}^{(2)}\right]^{2} \ell_{k}^{(1)}, \quad k=1,2,3, \\
& \beta_{1}=\left[a_{0}^{(1)}\right]^{2} a_{1}^{(2)} \ell_{k}^{(2)}+a_{1}^{(1)}\left[a_{0}^{(2)}\right]^{2} \ell_{k}^{(1)}, \quad k=1,2,3, \\
& \gamma_{1}=2\left[a_{0}^{(1)} a_{0}^{(2)}\right]^{2} b_{1}, \\
& N=a_{0}^{(1)} \ell_{k}^{(2)}+a_{0}^{(2)} \ell_{k}^{(1)}, \quad k=1,2,3, \\
& N_{b}=2 a_{0}^{(1)} a_{0}^{(2)} b_{1} .
\end{aligned}
$$


Note that parameters in (40c) and (40e) include the constant $b_{1}$, which takes into account the thermal resistance at the interface $\partial \Omega^{(1,2)}$. The homogenization scheme in (34)-(37) can be continuously repeated in order to derive the homogenized heat equations of higher asymptotic orders $\epsilon^{n}, n \geq 2$.

Once the effective parameters $\left\langle a_{0}\right\rangle$ and $\left\langle a_{0}\right\rangle$ have been determined, we obtain the homogenized thermal conductivity $\langle k\rangle$ and the homogenized heat flux $\langle q\rangle$ of the order $\epsilon^{0}$ in the form

$$
\begin{aligned}
& \langle k\rangle=\left\langle a_{0}\right\rangle+T_{0}\left\langle a_{1}\right\rangle, \\
& \langle q\rangle=-\left[\left\langle a_{0}\right\rangle+T_{0}\left\langle a_{1}\right\rangle\right] \frac{\partial T_{0}}{\partial \eta_{k}}, \quad k=1,2,3 .
\end{aligned}
$$

In the case of $a_{1}^{(1)}=a_{1}^{(2)}=0$ the effective thermal conductivity $\langle k\rangle=\left\langle a_{0}\right\rangle$ becomes

$$
\left\langle a_{0}\right\rangle=\frac{a_{0}^{(1)} a_{0}^{(2)} \ell}{a_{0}^{(1)} \ell^{(2)}+a_{0}^{(2)} \ell^{(1)}+2 a_{0}^{(1)} a_{0}^{(2)} b_{1}},
$$

which is temperature-independent. The result in (42) shows some analogies to the effective elastic properties of composites with imperfect bonding which have been obtained by Topol [45]. In the case of the absence of thermal resistance, $b_{1}=0,(42)$ reduces to

$$
\left\langle a_{0}\right\rangle=\frac{a_{0}^{(1)} a_{0}^{(2)} \ell}{a_{0}^{(1)} \ell^{(2)}+a_{0}^{(2)} \ell^{(1)}} .
$$

The form (43) for effective parameter in linear problems is well known from different problems in the field of mechanics. For example, Andrianov et al. [11] obtained a similar form for the effective elastic material parameters for wave propagation in layered composite for three-dimensional problems with large volume fractions of the inclusions. Zhang et al. [14] obtained this result in (43) as well as results for the effective thermal parameters of higher asymptotic orders.

\subsection{Heat diffusion in a layered composite}

The asymptotic homogenization of a layered composite is a well studied topic. Nevertheless, this relatively simple case of a layered material is useful to illustrate and to highlight the different features of the effective heat equation which has been obtained by the homogenization technique. Figure 4 shows a layered composite with the unit cell length $\ell$. The material is considered to consist of to alternating layers $\Omega^{(1)}$ and $\Omega^{(2)}$ with the lengths $\ell^{(1)}$ and $\ell^{(2)}$, respectively, in the $\mathbf{E}_{1}$-direction, so that $\ell^{(1)}+\ell^{(2)}=\ell$. In such case the governing heat equation can be derived from (38), which then takes the form

$$
\left\langle a_{0}\right\rangle \frac{\partial^{2} T_{0}}{\partial \eta_{1}^{2}}+\left\langle a_{1}\right\rangle\left[\left(\frac{\partial T_{0}}{\partial \eta_{1}}\right)^{2}+T_{0} \frac{\partial^{2} T_{0}}{\partial \eta_{1}^{2}}\right]+O\left(\epsilon^{1}\right)=\left\langle\rho_{p}\right\rangle \frac{\partial T_{0}}{\partial t}
$$

While (38) is valid for large size of the inclusions, i.e., $\ell_{k}^{(1)} \rightarrow \ell_{k}$, where $k=1,2,3$, Eq. (44) is valid for arbitrary thicknesses of the layers. 

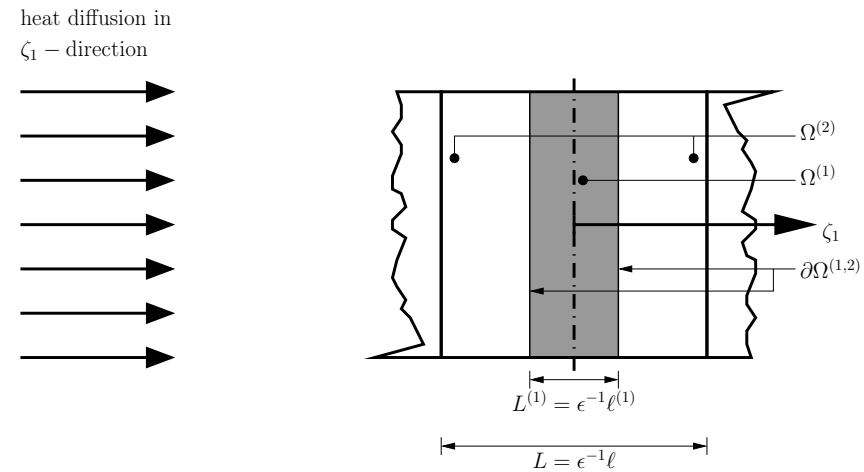

Figure 4: One-dimensional heat diffusion in a layered composite. The material is considered to consist of to alternating layers $\Omega^{(1)}$ and $\Omega^{(2)}$ with the lengths $L^{(1)}$ and $L^{(2)}$, respectively, in the $\mathbf{E}_{1}$-direction in terms of the fast coordinate variable $\zeta_{1}$, so that $L^{(1)}+L^{(2)}=L$, where $L$ is the total length of a single unit cell.

\subsection{Numerical examples}

We present two numerical examples to illustrate the different features of the herein derived homogenized heat equation. In the first example, we study a one-dimensional heat-diffusion problem in a layered composite, in which we contrast the results of the homogenized heat equation and the results of the heterogeneous original problem. In the second example, we study two-dimensional heat diffusion in a quadratic plate. The different results illustrate the influences of the temperature-dependent thermal conductivity of the constituents and varying interfacial conditions on the heat propagation in the plate. In both examples, we apply the finite difference method to treat the heat equations (38) and (44). Such method is described in detail in works such as [46], and the details of the herein applied form of such method are briefly summed up in Appendix B.

Heat diffusion in a layered composite. In the present example we consider a composite which consists of two alternating layers $\Omega^{(1)}$ and $\Omega^{(2)}$. The properties of $\Omega^{(1)}$ are based on the parameters of austenitic steel (Kh18N10T), and the properties of $\Omega^{(2)}$ are based on the parameters of aluminum 99.99, and the specific values of these parameters are cited from [47] for $293.14 \mathrm{~K}$, and they are as follows:

\begin{tabular}{||l|l||l|l||}
\hline \hline constituent & & $\Omega^{(1)}$ & $\Omega^{(2)}$ \\
\hline \hline length & $\ell_{1}^{(i)}$ & $\ell_{1}^{(1)}=0.5 \ell$ & $\ell_{1}^{(2)}=0.5 \ell$ \\
\hline thermal conductivity & $a_{0}^{(i)}\left[\mathrm{W} \mathrm{m}^{-1} \mathrm{~K}^{-1}\right]$ & 14.5 & 238 \\
\hline mass density & $\rho^{(i)}\left[\mathrm{kg} \mathrm{m}^{-3}\right]$ & 7900 & 2700 \\
\hline specific heat capacity & $c_{p}^{(i)}\left[\mathrm{J} \mathrm{kg}^{-1} \mathrm{~K}^{-1}\right]$ & 470 & 945 \\
\hline \hline
\end{tabular}

The thermal parameters are taken to be temperature-independent, $a_{1}^{(i)}=0$, and the thermal resistance at the interface $\partial \Omega^{(1,2)}$ is neglected, $b_{1}=0$. We consider a layered composite of the length $L=0.1 \mathrm{~m}$ in the direction $\mathbf{E}_{1}$ of heat diffusion, $x_{1} \in[0, L]$. The length of one unit cell is taken to be $\ell$, and both constituents to have the same thickness of $\ell_{1}^{(1)}=\ell_{1}^{(2)}=\ell / 2$. Heat diffusion analyzed by the application of the finite difference method. Therefore $L$ is subdivided into 100 


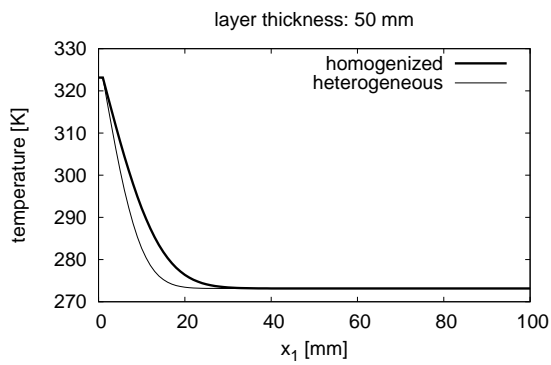

(a)

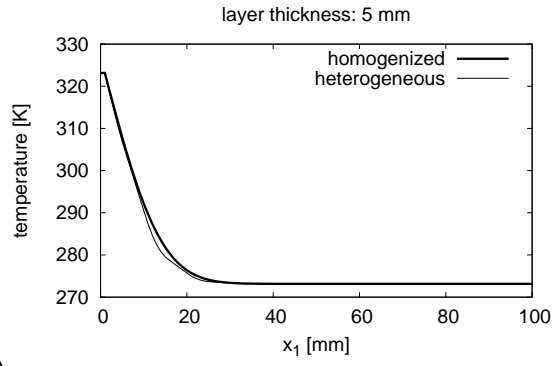

(c)

Figure 5: One-dimensional heat diffusion in a composite with two alternating layers $\Omega^{(1)}$ and $\Omega^{(1)}$.

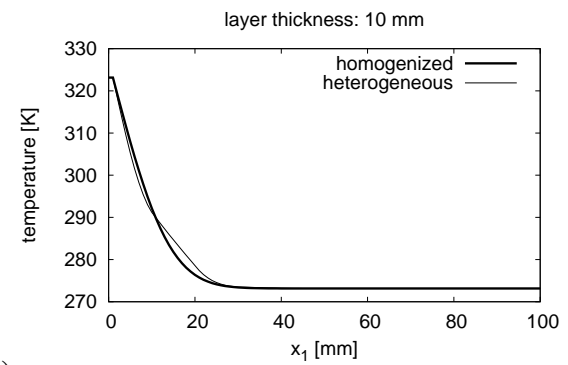

(b)

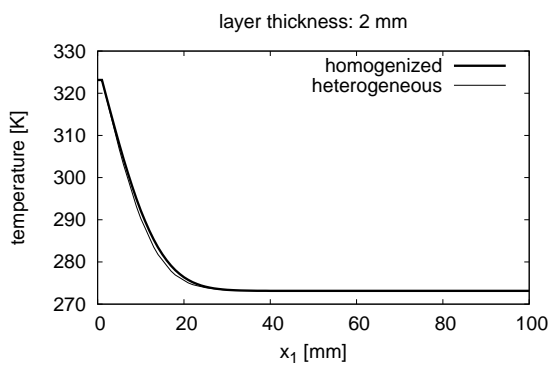

(d)

intervals, so that all 101 noted are located at $x_{1}=N \mathrm{~mm}$, where $N=0,1,2, \ldots, 101$. At all times $t$, the nodes for $N=1,2$ have the temperature $323.15 \mathrm{~K}$, and the nodes for $N=100,101$ have the temperature $273.15 \mathrm{~K}$. At time $t=0$ all further nodes have the temperature $273.15 \mathrm{~K}$. We want to contrast the solutions for the heat distribution at time $t=120 \mathrm{~s}$ (in $24 \cdot 10^{3} \mathrm{steps}$ ) in the material four different thicknesses for $\ell_{1}^{(1)}=\ell_{2}^{(1)}$ : (a) $50 \mathrm{~mm}$, (b) $10 \mathrm{~mm}$, (c) $5 \mathrm{~mm}$, and (d) $2 \mathrm{~mm}$. Figure 5 compares these cases to the homogenized solution of the order $O\left(\epsilon^{0}\right)$ in (44). In panel (a) we find that heat diffusion just took place in constituent $\Omega^{(1)}$. In the then following panels the thickness decreases, and the solution for the heterogeneous materials comes closer to the homogenized solution. Although the solution for the heterogeneous materials and the homogenized solution in panel (d) are pretty close together, we still find a small but visible difference between these solutions. If we would consider a heterogeneous material with a finer microstructure, then we would have to apply a finer mesh, and therefore larger computing times.

Nonlinear heat diffusion in a quadratic plate. In the present example we consider twodimensional heat diffusion in a quadratic plate with the side lengths $L=0.1 \mathrm{~m}$, so that $x_{1} \in[0, L]$, $x_{2} \in[0, L]$, and $\frac{\partial T}{\partial x_{3}}=0$. The microstructure consists of quadratic unit cells of the side length $\ell$, where $\ell \ll L$, with quadratic inclusions $\Omega^{(1)}$ of the side lengths $\ell_{1}^{(1)}=\ell_{2}^{(1)}=0.95 \ell$. The governing heat Eq. is given by (38). The material properties of constituent $\Omega^{(1)}$ are based on properties of copper 99.99 , and the properties of constituent $\Omega^{(2)}$ are based on the properties of 
aluminum 99.99. These properties are taken from [47], and they are as follows:

\begin{tabular}{||l|l||l|l||}
\hline \hline constituent & & $\Omega^{(1)}$ & $\Omega^{(2)}$ \\
\hline \hline length & $\ell^{(i)}$ & $\ell_{k}^{(1)}=0.95 \ell$ & $\ell_{k}^{(1)}=0.05 \ell$ \\
\hline thermal conductivity & $a_{0}^{(i)}\left[\mathrm{W} \mathrm{m}^{-1} \mathrm{~K}^{-1}\right]$ & 401 & 238 \\
\hline mass density & $\rho^{(i)}\left[\mathrm{kg} \mathrm{m}^{-3}\right]$ & 8960 & 2700 \\
\hline specific heat capacity & $c_{p}^{(i)}\left[\mathrm{J} \mathrm{kg}^{-1} \mathrm{~K}^{-1}\right]$ & 385 & 945 \\
\hline \hline
\end{tabular}

The plate is subdivided into $21 \times 21$ nodes. At all times $t$, the nodes located at the edges $x_{1}=L$ and $x_{2}=L$ have the temperature $273.13 \mathrm{~K}$, and all other edge nodes have the temperature $373.13 \mathrm{~K}$. At time $t=0$ all other nodes have the temperature $273.13 \mathrm{~K}$. The temperature distribution for a later time $t=4 \mathrm{~s}$ is illustrated in Fig. 6 .

- The panels (a)-(c) of Fig. 6 illustrate the heat diffusion for temperature-independent thermal parameters, $\left\langle a_{1}\right\rangle=0$, and different values of the thermal resistance. Panel (a) is for the absence of any thermal resistance at $\partial \Omega^{(1,2)}, b_{1}=0$, panel (b) is for $b_{1}=0.001 \mathrm{~W}^{-1} \mathrm{~K}$, and panel (c) is for $b_{1}=0.01 \mathrm{~W}^{-1} \mathrm{~K}$. These panels illustrate how increasing the values for $b_{1}$ slow down the diffusion of heat in the plate.

- For the bottom three panels (d)-(f) of Fig. 6 we assume the total absence of any thermal resistance at the interface $\partial \Omega^{(1,2)}$, so that $b_{1}=0$. We take the thermal conductivity of constituent $\Omega^{(1)}$ to be temperature-dependent, and therefore we define the parameter $a_{1}^{(1)}$ relative to $a_{0}^{(1)}$,

$$
a_{1}^{(1)}=M / \mathrm{K} a_{0}^{(1)} .
$$

For negative values for $M$, the values for the thermal conductivity $\kappa^{(1)}$ decrease with increasing temperatures. Such decrease of the thermal conductivity is usual for many metals in the considered temperature range [47]. Panel (d) is for $M=-1 / 1000$, panel (e) is for $M=-1 / 500$, and panel (f) is for $M=-1 / 400$. These panels show the change in the temperature diffusion when the thermal conductivity decreases with rising temperatures.

\section{Low volume fraction of the inclusions: using of three-phase model}

In the case of low volume fractions of the inclusions, $\frac{v^{(1)}}{v} \rightarrow 0$, we will apply the three-phase model in order to obtain the effective thermal properties of the composite. The three-phase model is also denoted as the self-consistent approximation, and it was proposed by Hershey [1] and Kerner [3], and then later further developed by Kröner [4], van der Poel [6], and Hill [2]. The theory behind this approach is explained, for example, in Christensen \& Lo [19] and Christensen [21]. The application of the three-phase model for the asymptotic analysis of heat conduction problems with small inclusions has been applied in different works, for example in [20]. In this approach, the original problem is replaced by a unit cell which contains one single inclusion $\Omega^{(1)}$, the first phase, which is surrounded by the matrix $\Omega^{(2)}$, the second phase. This inclusionmatrix part is surrounded by a third phase $\Omega^{(3)}$ with the same effective and yet unknown thermal properties as the composite on the macro-scale. By introducing a third phase $\Omega^{(3)}$, we also have to consider the interaction between the matrix and the third phase at their common interface $\partial \Omega^{(2,3)}$. At this interface we do not consider any thermal resistance, so that the conjugate conditions can be stated as follows: 
(a)

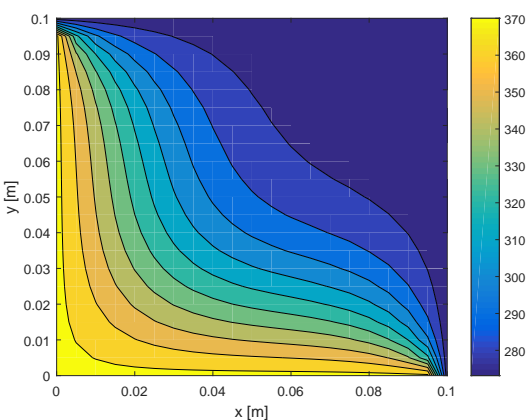

(c)

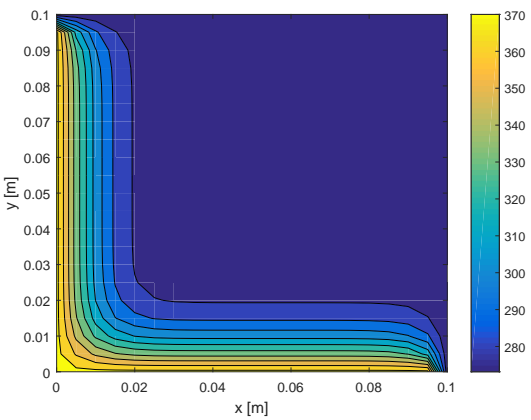

(e)

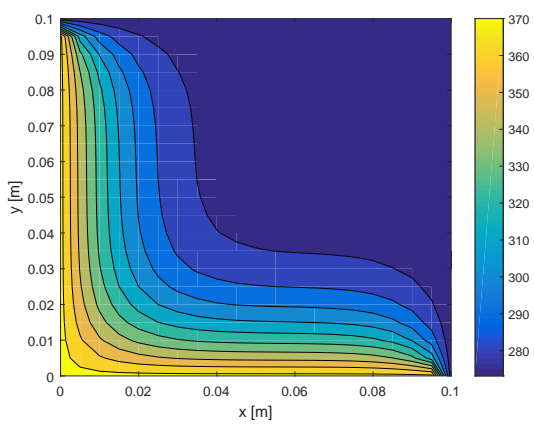

(b)

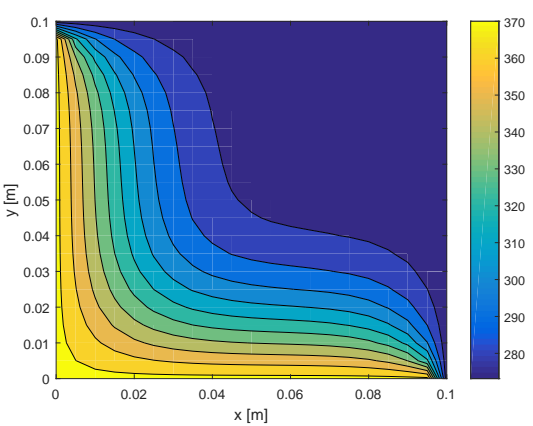

(d)

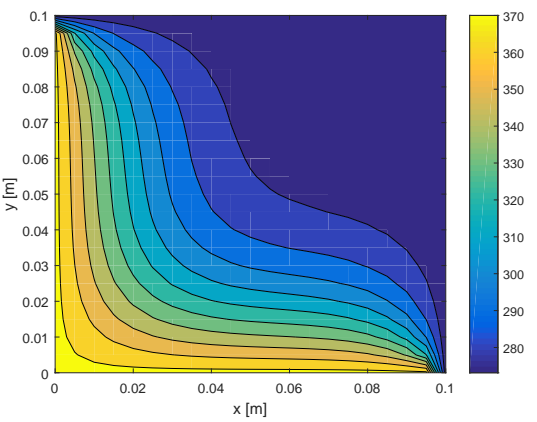

(f)

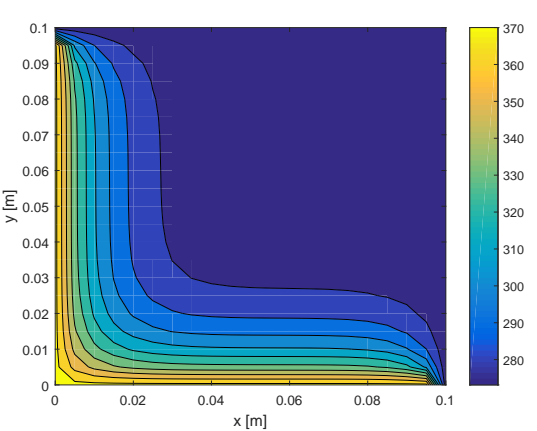

Figure 6: Two-dimensional heat diffusion in a quadratic plate: The top three panels (a)-(c) illustrate the heat diffusion for temperature-independent thermal parameters and different values of the thermal resistance. Panel (a) is for $b_{1}=0$, panel (b) is for $b_{1}=0.001 \mathrm{~W}^{-1} \mathrm{~K}$, and (c) is for $b_{1}=0.01 \mathrm{~W}^{-1} \mathrm{~K}$. The bottom three panels (d)-(f) show the heat distribution in the plate for temperature-dependent thermal conductivities of the inclusion.

- Equality of the heat flux in $\partial \Omega^{(2,3)}$ : Analogously to (46), we obtain

$$
\begin{aligned}
& \left\{a_{0}^{(2)}\left(\frac{\partial T_{n-1}^{(2)}}{\partial n_{\eta}}+\frac{\partial T_{n}^{(2)}}{\partial n_{\zeta}}\right)+a_{1}^{(2)}\left[\sum_{m=0}^{n-1} T_{m}^{(1)}\left(\frac{\partial T_{n-m-1}^{(2)}}{\partial n_{\eta}}+\frac{\partial T_{n-m}^{(2)}}{\partial n_{\zeta}}\right)\right]\right. \\
& \left.=a_{0}^{(3)}\left(\frac{\partial T_{n-1}^{(3)}}{\partial n_{\eta}}+\frac{\partial T_{n}^{(3)}}{\partial n_{\zeta}}\right)+a_{1}^{(3)}\left[\sum_{m=0}^{n-1} T_{m}^{(3)}\left(\frac{\partial T_{n-m-1}^{(3)}}{\partial n_{\eta}}+\frac{\partial T_{n-m}^{(3)}}{\partial n_{\zeta}}\right)\right]\right\}\left.\right|_{\partial \Omega^{(2,3)}}
\end{aligned}
$$


- Temperature difference in $\partial \Omega^{(2,3)}:$ In absence of any thermal resistance, the temperatures are equal at $\partial \Omega^{(2,3)}$.

$$
\left.\left\{\left(T_{n}^{(2)}=T_{n}^{(3)}\right)\right\}\right|_{\partial \Omega^{(2,3)}}
$$

In the following we will apply the three-phase model to analyze two different problems, which are illustrated in Fig. 2:

1. In the first case we analyze the effective properties of a composite in which the inclusions are arranged in a regular pattern of parallel cylindrical fibers. Heat diffusion is studied in the directions perpendicular to the fiber orientation.

2. In the second case, the inclusions are taken to have spherical shapes.

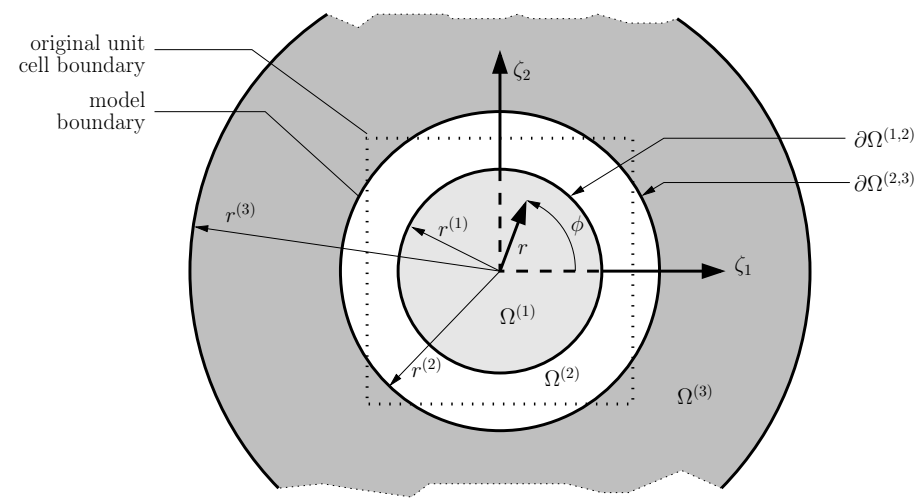

Figure 7: Three-phase model: A single unit cell of the composite. In this approach, the original problem is replaced by a unit cell which contains one single inclusion $\Omega^{(1)}$, the first phase, which is surrounded by the matrix $\Omega^{(2)}$, the second phase. This inclusion-matrix part is surrounded by a third phase $\Omega^{(3)}$ with the same effective properties as the composite on the macro-scale.

In this three-phase model, the original problems are studies by the application of an asymptotic model in which the original inclusion-matrix cell is replaced by a cylindrical cell in the case of parallel fibers, and by a spherical cell in the case of spherical inclusions (see Fig. 7). Such a modeling cell consists of the inclusion $\Omega^{(1)}$ in its center, a surrounding matrix phase $\Omega^{(2)}$. This inclusion-matrix part is surrounded by a third phase $\Omega^{(3)}$ with the same effective properties as the composite on the macro-scale. Such replacement has been applied in different works, and it is based on the zero-order approximation of the boundary shape perturbation method (see, for example, Guz \& Nemish [48] and Kalamkarov et al. [49]).

\subsection{Three-phase model applied to a composite with fiber inclusions}

We consider an array of parallel fibers in the matrix, which are oriented in the $\mathbf{E}_{3}$-direction. The centers of the fibers form a square lattice, and we assume heat diffusion in the $\mathbf{E}_{1}-\mathbf{E}_{2}$-plane perpendicular to the fiber orientation. Because $\frac{\partial T^{(i)}}{\partial \eta_{3}}=\frac{\partial T^{(i)}}{\partial \zeta_{3}}=0$, the considered boundary value problem can be reduced to a two-dimensional problem. The fibers have a circular cross-sectional area with the radius $r^{(1)}$ in the notation of the fast coordinate variables. 
It is now more convenient to consider this problem in a cylindrical coordinate system which is defined by the three base unit vectors $\left\{\mathbf{E}_{r_{c}}, \mathbf{E}_{\phi}, \mathbf{E}_{\zeta_{3}}\right\}$, and therefore we replace the Cartesian fast coordinate variables in $\zeta$ by cylindrical fast coordinate variables $\zeta_{c}$ with the elements $\left\{r, \phi, \zeta_{3}\right\}$. These coordinate systems are related via

$$
\begin{array}{ll}
\zeta_{1}=r \cos \phi, & r=\sqrt{\zeta_{1}^{2}+\zeta_{2}^{2}}, \\
\zeta_{2}=r \sin \phi, & \phi=\operatorname{atan} 2\left(\zeta_{2}, \zeta_{1}\right),
\end{array}
$$

where $r \geq 0$ and $0 \leq \phi \leq 2 \pi$.

We replace the previously applied notation of correction term $T_{n}^{(i)}(\boldsymbol{\eta}, \boldsymbol{\zeta}, t)$ of the temperature field by a notation in which we apply the cylindrical coordinates:

$$
\bar{T}_{n}^{(i)}\left(\boldsymbol{\eta}, \boldsymbol{\zeta}_{c}, t\right)=T_{n}^{(i)}(\boldsymbol{\eta}, \boldsymbol{\zeta}, t), \quad i=1,2,3 .
$$

The conjugate conditions (33) for the center and the outer boundaries of the original unit cell are replaced by the two conditions at the center of the cylindrical cell and at $r \rightarrow \infty$ (see, for example, [20]),

$$
\begin{aligned}
& \left.\left\{\bar{T}_{n}^{(1)}=0\right\}\right|_{r \rightarrow 0}, \\
& \left.\left\{\frac{\partial \bar{T}_{n}^{(3)}}{\partial r}=0\right\}\right|_{r \rightarrow \infty},
\end{aligned}
$$

where $n=1,2,3, \ldots$. Substituting $n=1$ into the heat equation (27), we obtain the following equation for the first correction term $\bar{T}_{1}^{(i)}$ of the temperature in the three constituents $\Omega^{(i)}$,

$$
\frac{\partial^{2} \bar{T}_{1}^{(i)}}{\partial \zeta_{1}^{2}}+\frac{\partial^{2} \bar{T}_{1}^{(i)}}{\partial \zeta_{2}^{2}}=0, \quad i=1,2,3
$$

Both $\zeta_{1}$ and $\zeta_{2}$ are fast Cartesian coordinates, and $\bar{T}_{1}^{(i)}$ is now defined in terms of the cylindrical coordinates $r$ and $\phi$. Therefore in (51) we apply the derivatives with respect to $\zeta_{1}$ and $\zeta_{2}$ in the forms

$$
\begin{aligned}
& \frac{\partial}{\partial \zeta_{1}}=\cos \phi \frac{\partial}{\partial r}-\frac{\sin \phi}{r} \frac{\partial}{\partial \phi} \\
& \frac{\partial}{\partial \zeta_{2}}=\sin \phi \frac{\partial}{\partial r}+\frac{\cos \phi}{r} \frac{\partial}{\partial \phi}
\end{aligned}
$$

so that (51) becomes

$$
\frac{\partial^{2} \bar{T}_{1}^{(i)}}{\partial r^{2}}+\frac{1}{r} \frac{\partial \bar{T}_{1}^{(i)}}{\partial r}+\frac{1}{r^{2}} \frac{\partial^{2} \bar{T}_{1}^{(i)}}{\partial \phi^{2}}=0, \quad i=1,2,3
$$

We now have to choose ansatzes which are capable to satisfy the conditions (50) and (53), and we choose

$$
\bar{T}_{1}^{(i)}=\frac{\partial T_{0}}{\partial n_{\eta}}\left[c_{1}^{(i)} r+c_{2}^{(i)} r^{-1}\right], \quad i=1,2,3,
$$


where $\frac{\partial T_{0}}{\partial n_{\eta}}=\frac{\partial T_{0}}{\partial \eta_{1}} \cos \phi+\frac{\partial T_{0}}{\partial \eta_{2}} \sin \phi$ is the directional derivative in radial direction $\mathbf{n}=\mathbf{E}_{1} \cos \phi+$ $\mathbf{E}_{2} \sin \phi$, normal to the interfaces of the three constituents. The six parameters $c_{1}^{(i)}$ and $c_{2}^{(i)}$ for $i=1,2,3$ are determined from the two conjugate conditions in (50), which immediately give $c_{2}^{(1)}=c_{1}^{(3)}=0$, and from the four conjugate conditions at the interfaces $\partial \Omega^{(1,2)}$ and $\partial \Omega^{(2,3)}$. Specifically, the conjugate conditions at the interface $\partial \Omega^{(1,2)}$ are given in Eqs. (28) and (29), and for the interface $\partial \Omega^{(2,3)}$ in (46) and (47), and for the here discussed boundary value problem these conjugate conditions take the following forms for the $\epsilon^{0}$ order considerations:

- Equality of the heat flux at the interface $\partial \Omega^{(1,2)}$ : From (28) we obtain

$$
\left[a_{0}^{(1)}+a_{1}^{(1)} T_{0}\right]\left(1+c^{(1)}\right)=\left[a_{0}^{(2)}+a_{1}^{(2)} T_{0}\right]\left(1+c_{1}^{(2)}-c_{2}^{(2)}\left[r^{(1)}\right]^{-2}\right) .
$$

- Temperature difference at the interface $\partial \Omega^{(1,2)}$ : From (29) we obtain

$$
\pm \epsilon\left[\left(c_{1}^{(2)}-c^{(1)}\right) r^{(1)}+c_{2}^{(2)}\left[r^{(1)}\right]^{-1}\right]=b_{1}\left[a_{0}^{(1)}+a_{1}^{(1)} T_{0}\right]\left(1+c^{(1)}\right) .
$$

- Equality of the heat flux at the interface $\partial \Omega^{(2,3)}$ : From (46) we obtain

$$
\left[a_{0}^{(2)}+a_{1}^{(2)} T_{0}\right]\left(1+c_{1}^{(2)}-c_{2}^{(2)}\left[r^{(2)}\right]^{-2}\right)=\left[a_{0}^{(3)}+a_{1}^{(3)} T_{0}\right]\left(1-c_{2}^{(3)}\left[r^{(2)}\right]^{-2}\right) .
$$

- Temperature difference at the interface $\partial \Omega^{(2,3)}$ : From (47) we obtain

$$
c_{1}^{(2)} r^{(2)}+c_{2}^{(2)}\left[r^{(2)}\right]^{-1}=c^{(3)}\left[r^{(2)}\right]^{-1} .
$$

For the herein considered two-dimensional problem in terms of the fast Cartesian coordinates, the left side of heat Eq. (27) takes the following form,

$$
\begin{aligned}
H^{(i)} & =\sum_{k=1}^{2} a_{0}^{(i)}\left(\frac{\partial^{2} T_{0}}{\partial \eta_{k}^{2}}+\frac{\partial^{2} T_{1}^{(i)}}{\partial \eta_{k} \partial \zeta_{k}}\right)+a_{1}^{(i)}\left(\frac{\partial T_{0}}{\partial \eta_{k}}+\frac{\partial T_{1}^{(i)}}{\partial \zeta_{k}}\right)^{2} \\
& +a_{1}^{(i)} T_{0}\left(\frac{\partial^{2} T_{0}}{\partial \eta_{k}^{2}}+\frac{\partial^{2} T_{1}^{(i)}}{\partial \eta_{k} \partial \zeta_{k}}\right)
\end{aligned}
$$

where $H^{(i)}=H^{(i)}(\boldsymbol{\eta}, \zeta)$. Recalling $\frac{\partial^{2}}{\partial \eta_{1} \partial \eta_{2}}=0$ and applying the change from fast Cartesian coordinate variables to cylindrical coordinate variables in (49), Eq. (59) takes the following form after substitution of the ansatzes (54),

$$
\begin{aligned}
\bar{H}^{(i)} & =\sum_{k=1}^{2} a_{0}^{(i)}\left(\frac{\partial^{2} T_{0}}{\partial \eta_{k}^{2}}+\frac{\partial^{2} \bar{T}_{1}^{(i)}}{\partial \eta_{k} \partial \zeta_{k}}\right)+a_{1}^{(i)}\left(\frac{\partial T_{0}}{\partial \eta_{k}}+\frac{\partial \bar{T}_{1}^{(i)}}{\partial \zeta_{k}}\right)^{2} \\
& +a_{1}^{(i)} T_{0}\left(\frac{\partial^{2} T_{0}}{\partial \eta_{k}^{2}}+\frac{\partial^{2} \bar{T}_{1}^{(i)}}{\partial \eta_{k} \partial \zeta_{k}}\right)
\end{aligned}
$$

were $\bar{H}^{(i)}=\bar{H}^{(i)}\left(\boldsymbol{\eta}, \boldsymbol{\zeta}_{c}\right)$. The specific forms of the derivatives in (60) are presented in Appendix 


$$
\langle\cdot\rangle=\frac{1}{A} \iint_{A}(\cdot) r \mathrm{~d} r \mathrm{~d} \phi,
$$

413

over (60) we obtain

$$
\begin{aligned}
& \frac{1}{A}\left\{\int_{0}^{2 \pi} \int_{0}^{r^{(1)}} \bar{H}^{(1)} r \mathrm{~d} r \mathrm{~d} \phi+\int_{0}^{2 \pi} \int_{r^{(1)}}^{r^{(2)}} \bar{H}^{(2)} r \mathrm{~d} r \mathrm{~d} \phi+\int_{0}^{2 \pi} \int_{r^{(2)}}^{r^{(3)}} \bar{H}^{(3)} r \mathrm{~d} r \mathrm{~d} \phi\right] \\
= & \frac{1}{A}\left\{a_{0}^{(1)} A^{(1)} \bar{\alpha}_{0}^{(1)}+a_{0}^{(2)} A^{(2)} \bar{\alpha}_{0}^{(2)}+\underline{\underline{a_{0}^{(3)} A^{(3)} \bar{\alpha}_{0}^{(2)}}}\right. \\
+ & a_{1}^{(1)} A^{(1)} \bar{\alpha}_{1}^{(1)}+a_{1}^{(2)} A^{(2)} \bar{\alpha}_{1}^{(2)}+a_{1}^{(3)} A^{(3)} \bar{\alpha}_{1}^{(3)} \\
+ & \left.a_{1}^{(1)} A^{(1)} T_{0} \bar{\alpha}_{0}^{(1)}+a_{1}^{(2)} A^{(2)} T_{0} \bar{\alpha}_{0}^{(2)}+\underline{\underline{a_{1}^{(3)} A^{(3)} T_{0} \bar{\alpha}_{0}^{(3)}}}\right\},
\end{aligned}
$$

where we have applied the abbreviations

$$
\begin{aligned}
& \bar{\alpha}_{0}^{(1)}=\left(c_{1}^{(1)}+1\right) \alpha_{0}^{(3)}, \\
& \bar{\alpha}_{0}^{(2)}=\left(c_{1}^{(2)}+1\right) \alpha_{0}^{(3)}, \\
& \bar{\alpha}_{0}^{(3)}=\left[\frac{\partial^{2} T_{0}}{\partial \eta_{1}^{2}}+\frac{\partial^{2} T_{0}}{\partial \eta_{2}^{2}}\right],
\end{aligned}
$$

and

$$
\begin{aligned}
& \bar{\alpha}_{1}^{(1)}=\left(c_{1}^{(1)}+1\right)^{2}\left[\left(\frac{\partial T_{0}}{\partial \eta_{1}}\right)^{2}+\left(\frac{\partial T_{0}}{\partial \eta_{2}}\right)^{2}\right], \\
& \bar{\alpha}_{1}^{(2)}=\left[\left(c_{1}^{(2)}+1\right)^{2}+\frac{\left[c_{2}^{(2)}\right]^{2}}{2\left[r^{(1)} r^{(2)}\right]^{2}}\right]\left[\left(\frac{\partial T_{0}}{\partial \eta_{1}}\right)^{2}+\left(\frac{\partial T_{0}}{\partial \eta_{2}}\right)^{2}\right], \\
& \bar{\alpha}_{1}^{(3)}=\left[\frac{\left[c_{2}^{(3)}\right]^{2}}{2\left[r^{(2)} r^{(3)}\right]^{2}}+\underline{1}\right]\left[\left(\frac{\partial T_{0}}{\partial \eta_{1}}\right)^{2}+\left(\frac{\partial T_{0}}{\partial \eta_{2}}\right)^{2}\right] .
\end{aligned}
$$

$414 \quad$ In (62), $A=A^{(1)}+A^{(2)}+A^{(3)}=\pi\left[r^{(3)}\right]^{2}$ is the cross-sectional area of the unit cell, $A^{(1)}=\pi\left[r^{(1)}\right]^{2}$ 415 is the cross-sectional area of $\Omega^{(1)}, A^{(2)}=\pi\left(\left[r^{(2)}\right]^{2}-\left[r^{(1)}\right]^{2}\right)$ is the cross-sectional area of $\Omega^{(2)}$, 416 and $A^{(3)}=\pi\left(\left[r^{(3)}\right]^{2}-\left[r^{(2)}\right]^{2}\right)$ is the cross-sectional area of $\Omega^{(3)}$. Equation (62) is equal to the left ${ }_{417}$ hand side of the homogenized heat equation in the form

$$
\sum_{k=1}^{2}\left\{\left\langle a_{0}\right\rangle_{c} \frac{\partial^{2} T_{0}}{\partial \eta_{k}^{2}}+\left\langle a_{1}\right\rangle_{c}\left[\left(\frac{\partial T_{0}}{\partial \eta_{k}}\right)^{2}+T_{0} \frac{\partial^{2} T_{0}}{\partial \eta_{k}^{2}}\right]\right\},
$$


where $\left\langle a_{0}\right\rangle_{c}$ and $\left\langle a_{1}\right\rangle_{c}$ are the effective thermal parameters. The outer phase $\Omega^{(3)}$ of the unit cell has the same properties as overall thermal properties the composite, so that $\left\langle a_{0}\right\rangle_{c}=a_{0}^{(3)}$ and $\left\langle a_{1}\right\rangle_{c}=a_{1}^{(3)}$. If we now write "(65)=(62)", subtract the double underlined terms in (62) and (64) from both sides, and multiply both sides with $A$, then we arrive to an equation in the following form:

$$
\begin{aligned}
& \sum_{k=1}^{2}\left\{\left\langle a_{0}\right\rangle_{c} \frac{\partial^{2} T_{0}}{\partial \eta_{k}^{2}}+\left\langle a_{1}\right\rangle_{c}\left[\left(\frac{\partial T_{0}}{\partial \eta_{k}}\right)^{2}+T_{0} \frac{\partial^{2} T_{0}}{\partial \eta_{k}^{2}}\right]\right\} \\
= & \frac{1}{A^{(1)}+A^{(2)}}\left[a_{0}^{(1)} A^{(1)}\left(c_{1}^{(1)}+1\right)\left[\frac{\partial^{2} T_{0}}{\partial \eta_{1}^{2}}+\frac{\partial^{2} T_{0}}{\partial \eta_{2}^{2}}\right]\right. \\
+ & a_{0}^{(2)} A^{(2)}\left(c_{1}^{(2)}+1\right)\left[\frac{\partial^{2} T_{0}}{\partial \eta_{1}^{2}}+\frac{\partial^{2} T_{0}}{\partial \eta_{2}^{2}}\right] \\
+ & a_{1}^{(1)} A^{(1)}\left(c_{1}^{(1)}+1\right)^{2}\left[\left(\frac{\partial T_{0}}{\partial \eta_{1}}\right)^{2}+\left(\frac{\partial T_{0}}{\partial \eta_{2}}\right)^{2}\right] \\
+ & a_{1}^{(2)} A^{(2)}\left[\left(c_{1}^{(2)}+1\right)^{2}+\frac{\left[c_{2}^{(2)}\right]^{2}}{2\left[r^{(1)} r^{(2)}\right]^{2}}\right]\left[\left(\frac{\partial T_{0}}{\partial \eta_{1}}\right)^{2}+\left(\frac{\partial T_{0}}{\partial \eta_{2}}\right)^{2}\right] \\
+ & a_{1}^{(1)} A^{(1)}\left(c_{1}^{(1)}+1\right) T_{0}\left[\frac{\partial^{2} T_{0}}{\partial \eta_{1}^{2}}+\frac{\partial^{2} T_{0}}{\partial \eta_{2}^{2}}\right] \\
+ & \left.a_{1}^{(2)} A^{(2)}\left(c_{1}^{(2)}+1\right) T_{0}\left[\frac{\partial^{2} T_{0}}{\partial \eta_{1}^{2}}+\frac{\partial^{2} T_{0}}{\partial \eta_{2}^{2}}\right]\right\} .
\end{aligned}
$$

Because the radius $r^{(3)}$ of the unit cell is taken to be of infinite length in the asymptotic model, $r^{(3)} \rightarrow \infty$, the single underlined term in (64) has vanished. Note that due to the conjugate conditions at the interfaces the parameters $c_{1}^{(i)}$ and $c_{3}^{(i)}$ are also functions of $T_{0}$ and its derivatives. If we substitute $c_{1}^{(i)}$ and $c_{3}^{(i)}$ into (66), linearize the result and neglect all terms of higher order than in the effective heat equation, then we obtain the effective parameters by comparing the different terms on the left-hand sides and right-hand sides of (66).

- By comparing the factors of $\frac{\partial^{2} T_{0}}{\partial \eta_{1}^{2}}$ (or $\left.\frac{\partial^{2} T_{0}}{\partial \eta_{2}^{2}}\right)$, we obtain

$$
\left\langle a_{0}\right\rangle_{c}=a_{0}^{(2)} \frac{a_{0}^{(1)} \bar{r}_{+}+a_{0}^{(2)} \bar{r}_{-}+a_{0}^{(1)} a_{0}^{(2)} \hat{b}_{1} \bar{r}_{-}}{a_{0}^{(1)} \bar{r}_{-}+a_{0}^{(2)} \bar{r}_{+}+a_{0}^{(1)} a_{0}^{(2)} \hat{b}_{1} \bar{r}_{+}},
$$

$$
\text { where } \bar{r}_{+}=\left[r^{(2)}\right]^{2}+\left[r^{(1)}\right]^{2}, \bar{r}_{-}=\left[r^{(2)}\right]^{2}-\left[r^{(1)}\right]^{2} \text {, and } \hat{b}_{1}=b_{1} / r^{(1)} \text {. }
$$

- If we compare the factors of either $T_{0} \frac{\partial^{2} T_{0}}{\partial \eta_{1}}$ or $\left(\frac{\partial T_{0}}{\partial \eta_{1}}\right)^{2}$ on both sides of (66) and substitute (67), 
then we obtain

$$
\begin{aligned}
\left\langle a_{1}\right\rangle_{c} & =-\left\{\left(\left[a_{0}^{(1)}\right]^{2}+\left[a_{0}^{(2)}\right]^{2}\right) a_{1}^{(2)}\left[r^{(1)}\right]^{4}-2\left(\left[a_{0}^{(1)}\right]^{2}+\left[a_{0}^{(2)}\right]^{2}\right) a_{1}^{(2)}\left[r^{(2)}\right]^{4}\right. \\
& -8 a_{1}^{(1)}\left[a_{0}^{(2)}\right]^{2}\left[r^{(1)} r^{(2)}\right]^{2}+\left(\left[a_{0}^{(1)}\right]^{2}+\left[a_{0}^{(2)}\right]^{2}\right) a_{1}^{(2)}\left[r^{(1)} r^{(2)}\right]^{2} \\
& +\left(6\left[r^{(1)} r^{(2)}\right]^{2}-2\left[r^{(1)}\right]^{4}-4\left[r^{(2)}\right]^{4}\right) a_{0}^{(1)} a_{0}^{(2)} a_{1}^{(2)} \\
& +2\left(a_{0}^{(1)}\left[a_{0}^{(2)}\right]^{2}-\left[a_{0}^{(1)}\right]^{2} a_{0}^{(2)}\right) a_{1}^{(2)} \hat{b}_{1}\left[r^{(1)}\right]^{4} \\
& -4\left(a_{0}^{(1)}\left[a_{0}^{(2)}\right]^{2}+\left[a_{0}^{(1)}\right]^{2} a_{0}^{(2)}\right) a_{1}^{(2)} \hat{b}_{1}\left[r^{(2)}\right]^{4} \\
& +\left[a_{0}^{(1)} a_{0}^{(2)}\right]^{2} a_{1}^{(2)} \hat{b}_{1}^{2}\left(\left[r^{(1)}\right]^{4}-2\left[r^{(2)}\right]^{4}+\left[r^{(1)} r^{(2)}\right]^{2}\right) \\
& \left.+\left(2 a_{0}^{(1)}\left[a_{0}^{(2)}\right]^{2}+6\left[a_{0}^{(1)}\right]^{2} a_{0}^{(2)}\right) a_{1}^{(2)} \hat{b}_{1}\left[r^{(1)} r^{(2)}\right]^{2}\right\} \\
& /\left\{2 \left[a_{0}^{(1)}\left(\left[r^{(2)}\right]^{2}-\left[r^{(1)}\right]^{2}\right)+a_{0}^{(2)}\left(\left[r^{(2)}\right]^{2}+\left[r^{(1)}\right]^{2}\right)\right.\right. \\
& \left.\left.+a_{0}^{(1)} a_{0}^{(2)} \hat{b}_{1}\left(\left[r^{(2)}\right]^{2}+\left[r^{(1)}\right]^{2}\right)\right]^{2}\right\} .
\end{aligned}
$$

\subsection{Three-phase model applied to a composites with spherical inclusions}

We consider spherical inclusions with the radius $r^{(1)}$ in terms of the fast coordinate variables. This problem will be considered in a spherical coordinate system which is defined by the three base unit vectors $\left\{\mathbf{E}_{r}, \mathbf{E}_{\phi}, \mathbf{E}_{\theta}\right\}$, and therefore we replace the Cartesian fast coordinate variables in $\zeta$ by spherical fast coordinate variables $\zeta_{s}$ with the elements $\{r, \phi, \theta\}$,

$$
\begin{array}{llrl}
\zeta_{1} & =r \sin \theta \cos \phi, & r & =\sqrt{\zeta_{1}^{2}+\zeta_{2}^{2}+\zeta_{3}^{2}}, \\
\zeta_{2} & =r \sin \theta \sin \phi, & \phi & =\operatorname{atan} 2\left(\zeta_{2}, \zeta_{1}\right), \\
\zeta_{3} & =\cos \theta, & \theta & =\arccos \left(\zeta_{3} / \sqrt{\zeta_{1}^{2}+\zeta_{2}^{2}+\zeta_{3}^{2}}\right),
\end{array}
$$

where $r \geq 0,0 \leq \phi \leq 2 \pi$, and $0 \leq \theta \leq \pi$.

We replace the previously applied notation of correction term $T_{n}^{(i)}(\boldsymbol{\eta}, \boldsymbol{\zeta}, t)$ of the temperature field by a notation in which we apply the fast spherical coordinates:

$$
\hat{T}_{n}^{(i)}\left(\boldsymbol{\eta}, \zeta_{s}, t\right)=T_{n}^{(i)}(\boldsymbol{\eta}, \zeta, t), \quad i=1,2,3 .
$$

The conjugate conditions (33) for the center and the outer boundaries of the unit cell then take the forms (50), where now $r$ is the spherical radial coordinate. Substituting $n=1$ into the heat equation (27), we obtain the following equation for the first correction term $\hat{T}_{1}^{(i)}$ of the temperature in the three constituents $\Omega^{(i)}$,

$$
\frac{\partial^{2} \hat{T}_{1}^{(i)}}{\partial \zeta_{1}^{2}}+\frac{\partial^{2} \hat{T}_{1}^{(i)}}{\partial \zeta_{2}^{2}}+\frac{\partial^{2} \hat{T}_{1}^{(i)}}{\partial \zeta_{3}^{2}}=0, \quad i=1,2,3
$$

In (71) $\zeta_{1}, \zeta_{2}$ and $\zeta_{3}$ are fast Cartesian coordinates, and $\hat{T}_{1}^{(i)}$ is now defined in terms of the fast spherical coordinates $r, \theta$, and $\phi$. Therefore we apply the derivatives with respect to $\zeta_{1}$, $\zeta_{2}$, and 
$\zeta_{3}$ in the forms

$$
\begin{aligned}
& \frac{\partial}{\partial \zeta_{1}}=\cos \phi \sin \theta \frac{\partial}{\partial r}-\frac{\sin \phi}{r \sin \theta} \frac{\partial}{\partial \phi}+\frac{\cos \phi \cos \theta}{r} \frac{\partial}{\partial \theta} \\
& \frac{\partial}{\partial \zeta_{2}}=\sin \phi \sin \theta \frac{\partial}{\partial r}+\frac{\cos \phi}{r \sin \theta} \frac{\partial}{\partial \phi}+\frac{\sin \phi \cos \theta}{r} \frac{\partial}{\partial \theta} \\
& \frac{\partial}{\partial \zeta_{3}}=\cos \theta \frac{\partial}{\partial r}-\frac{\sin \theta}{r} \frac{\partial}{\partial \theta}
\end{aligned}
$$

so that (71) becomes

$$
\frac{\partial^{2} \hat{T}_{1}^{(i)}}{\partial r^{2}}+\frac{2}{r} \frac{\partial \hat{T}_{1}^{(i)}}{\partial r}+\frac{1}{r^{2} \sin ^{2} \theta} \frac{\partial^{2} \hat{T}_{1}^{(i)}}{\partial^{2} \phi}+\frac{\cos \theta}{r \sin \theta} \frac{\partial T_{1}^{(i)}}{\partial \theta}+\frac{1}{r^{2}} \frac{\partial^{2} T_{1}^{(i)}}{\partial \theta^{2}}=0
$$

where $i=1,2,3$. Ansatzes which satisfy (50) and (73) are

$$
T_{1}^{(i)}=\frac{\partial T_{0}}{\partial n_{\eta}}\left[c_{1}^{(i)} r+c_{2}^{(i)} r^{-2}\right], \quad i=1,2,3
$$

where $\frac{\partial T_{0}}{\partial n_{\eta}}=\left[\frac{\partial T_{0}}{\partial \eta_{1}} \sin \theta \cos \phi+\frac{\partial T_{0}}{\partial \eta_{2}} \sin \theta \sin \phi+\frac{\partial T_{0}}{\partial \eta_{3}} \cos \theta\right]$ is the directional derivative in radial direction $\mathbf{n}=\mathbf{E}_{1} \sin \theta \cos \phi+\mathbf{E}_{2} \sin \theta \sin \phi+\mathbf{E}_{3} \cos \theta$. As in the previous section, the six parameters $c_{1}^{(i)}$ and $c_{2}^{(i)}$ for $i=1,2,3$ are determined from the two conjugate conditions in (50), which immediately give $c_{2}^{(1)}=c_{1}^{(3)}=0$, from the four conjugate conditions at the interface $\partial \Omega^{(1,2)}$ in Eqs. (28) and (29), and for the interface $\partial \Omega^{(2,3)}$ in Eqs. (46) and (47):

- Equality of the heat flux at the interface $\partial \Omega^{(1,2)}$ : From (28) we obtain

$$
\left[a_{0}^{(1)}+a_{1}^{(1)} T_{0}\right]\left(1+c^{(1)}\right)=\left[a_{0}^{(2)}+a_{1}^{(2)} T_{0}\right]\left(1+c_{1}^{(2)}-2 c_{2}^{(2)}\left[r^{(1)}\right]^{-3}\right) .
$$

- Temperature difference at the interface $\partial \Omega^{(1,2)}$ : From (29) we obtain

$$
\pm \epsilon\left[\left(c_{1}^{(2)}-c^{(1)}\right) r^{(1)}+c_{2}^{(2)}\left[r^{(1)}\right]^{-2}\right]=b_{1}\left[a_{0}^{(1)}+a_{1}^{(1)} T_{0}\right]\left(1+c^{(1)}\right) .
$$

- Equality of the heat flux at the interface $\partial \Omega^{(2,3)}$ : From (46) we obtain

$$
\left[a_{0}^{(2)}+a_{1}^{(2)} T_{0}\right]\left(1+c_{1}^{(2)}-2 c_{2}^{(2)}\left[r^{(2)}\right]^{-3}\right)=\left[a_{0}^{(3)}+a_{1}^{(3)} T_{0}\right]\left(1-2 c_{2}^{(3)}\left[r^{(2)}\right]^{-3}\right) .
$$

- Temperature difference at the interface $\partial \Omega^{(2,3)}$ : From (47) we obtain

$$
c_{1}^{(2)} r^{(2)}+c_{2}^{(2)}\left[r^{(2)}\right]^{-2}=c^{(3)}\left[r^{(2)}\right]^{-2} .
$$

For the herein considered problem in terms of the Cartesian coordinates, the left side of (27) takes the following form,

$$
\begin{aligned}
H^{(i)} & =\sum_{k=1}^{3} a_{0}^{(i)}\left(\frac{\partial^{2} T_{0}}{\partial \eta_{k}^{2}}+\frac{\partial^{2} T_{1}^{(i)}}{\partial \eta_{k} \partial \zeta_{k}}\right)+a_{1}^{(i)}\left(\frac{\partial T_{0}}{\partial \eta_{k}}+\frac{\partial T_{1}^{(i)}}{\partial \zeta_{k}}\right)^{2} \\
& +a_{1}^{(i)} T_{0}\left(\frac{\partial^{2} T_{0}}{\partial \eta_{k}^{2}}+\frac{\partial^{2} T_{1}^{(i)}}{\partial \eta_{k} \partial \zeta_{k}}\right),
\end{aligned}
$$


over (80) we obtain

$$
\begin{aligned}
& \frac{1}{V}\left\{\int_{0}^{\pi} \int_{0}^{2 \pi} \int_{0}^{r^{(1)}} \hat{H}^{(1)} r^{2} \sin \theta \mathrm{d} r \mathrm{~d} \phi \mathrm{d} \theta+\int_{0}^{\pi} \int_{0}^{2 \pi} \int_{r^{(1)}}^{r^{(2)}} \hat{H}^{(2)} r^{2} \sin \theta \mathrm{d} r \mathrm{~d} \phi \mathrm{d} \theta\right. \\
& \left.+\int_{0}^{\pi} \int_{0}^{2 \pi} \int_{r^{(2)}}^{r^{(3)}} \hat{H}^{(3)} r^{2} \sin \theta \mathrm{d} r \mathrm{~d} \phi \mathrm{d} \theta\right\} \\
& =\frac{1}{V}\left\{a_{0}^{(1)} V^{(1)} \hat{\alpha}_{0}^{(1)}+a_{0}^{(2)} V^{(2)} \hat{\alpha}_{0}^{(2)}+\underline{\underline{a_{0}^{(3)} V^{(3)}}} \hat{\alpha}_{0}^{(3)}\right. \\
& +a_{1}^{(1)} V^{(1)} \hat{\alpha}_{1}^{(1)}+a_{1}^{(2)} V^{(2)} \hat{\alpha}_{1}^{(2)}+a_{1}^{(3)} V^{(3)} \hat{\alpha}_{1}^{(3)} \\
& \left.+a_{1}^{(1)} V^{(1)} T_{0} \hat{\alpha}_{0}^{(1)}+a_{1}^{(2)} V^{(2)} T_{0} \hat{\alpha}_{0}^{(2)}+\underline{\underline{a_{1}^{(3)} V^{(3)}}} T_{0} \hat{\alpha}_{0}^{(3)}\right\},
\end{aligned}
$$

where we have applied the abbreviations

$$
\begin{aligned}
& \hat{\alpha}_{0}^{(1)}=\left(c_{1}^{(1)}+1\right)\left[\frac{\partial^{2} T_{0}}{\partial \eta_{1}^{2}}+\frac{\partial^{2} T_{0}}{\partial \eta_{2}^{2}}+\frac{\partial^{2} T_{0}}{\partial \eta_{3}^{2}}\right], \\
& \hat{\alpha}_{0}^{(2)}=\left(c_{1}^{(2)}+1\right)\left[\frac{\partial^{2} T_{0}}{\partial \eta_{1}^{2}}+\frac{\partial^{2} T_{0}}{\partial \eta_{2}^{2}}+\frac{\partial^{2} T_{0}}{\partial \eta_{3}^{2}}\right], \\
& \hat{\alpha}_{0}^{(3)}=\left[\frac{\partial^{2} T_{0}}{\partial \eta_{1}^{2}}+\frac{\partial^{2} T_{0}}{\partial \eta_{2}^{2}}+\frac{\partial^{2} T_{0}}{\partial \eta_{3}^{2}}\right]
\end{aligned}
$$


and

$$
\begin{aligned}
& \hat{\alpha}_{1}^{(1)}=\left(c_{1}^{(1)}+1\right)^{2}\left[\left(\frac{\partial T_{0}}{\partial \eta_{1}}\right)^{2}+\left(\frac{\partial T_{0}}{\partial \eta_{2}}\right)^{2}+\left(\frac{\partial T_{0}}{\partial \eta_{3}}\right)^{2}\right] \\
& \hat{\alpha}_{1}^{(2)}=\left[\left(c_{1}^{(2)}+1\right)^{2}+\frac{4\left[c_{2}^{(2)}\right]^{2}}{5\left[r^{(1)} r^{(2)}\right]^{3}}\right]\left[\left(\frac{\partial T_{0}}{\partial \eta_{1}}\right)^{2}+\left(\frac{\partial T_{0}}{\partial \eta_{2}}\right)^{2}+\left(\frac{\partial T_{0}}{\partial \eta_{3}}\right)^{2}\right], \\
& \hat{\alpha}_{1}^{(3)}=\left[\frac{4\left[c_{2}^{(3)}\right]^{2}}{5\left[r^{(2)} r^{(3)}\right]^{3}}+\underline{1}\right]\left[\left(\frac{\partial T_{0}}{\partial \eta_{1}}\right)^{2}+\left(\frac{\partial T_{0}}{\partial \eta_{2}}\right)^{2}+\left(\frac{\partial T_{0}}{\partial \eta_{3}}\right)^{2}\right]
\end{aligned}
$$

${ }_{460}$ In (82), $V=V^{(1)}+V^{(2)}+V^{(3)}=\frac{4}{3} \pi\left[r^{(3)}\right]^{3}$ is the volume of the unit cell, $V^{(1)}=\frac{4}{3} \pi\left[r^{(1)}\right]^{3}$ is the

461

volume of $\Omega^{(1)}, V^{(2)}=\frac{4}{3} \pi\left(\left[r^{(2)}\right]^{3}-\left[r^{(1)}\right]^{3}\right)$ is the volume of $\Omega^{(2)}$, and $V^{(3)}=\frac{4}{3} \pi\left(\left[r^{(3)}\right]^{3}-\left[r^{(2)}\right]^{3}\right)$ is the volume of $\Omega^{(3)}$. Equation (82) is equal to the left hand side of the homogenized heat equation in the form

$$
\sum_{k=1}^{3}\left\{\left\langle a_{0}\right\rangle_{s} \frac{\partial^{2} T_{0}}{\partial \eta_{k}^{2}}+\left\langle a_{1}\right\rangle_{s}\left[\left(\frac{\partial T_{0}}{\partial \eta_{k}}\right)^{2}+T_{0} \frac{\partial^{2} T_{0}}{\partial \eta_{k}^{2}}\right]\right\}
$$

where $\left\langle a_{0}\right\rangle_{c}$ and $\left\langle a_{1}\right\rangle_{c}$ are the effective thermal parameters. The outer phase $\Omega^{(3)}$ of the unit cell has the same properties as overall thermal properties, so that $\left\langle a_{0}\right\rangle_{c}=a_{0}^{(3)}$ and $\left\langle a_{1}\right\rangle_{c}=a_{1}^{(3)}$. If we now write " $(85)=(82) "$, subtract the double underlined terms in (82) and (84) from both sides and multiply both sides with $V$, then we arrive to an equation in the following form:

$$
\begin{aligned}
& \sum_{k=1}^{3}\left\{\left\langle a_{0}\right\rangle_{s} \frac{\partial^{2} T_{0}}{\partial \eta_{k}^{2}}+\left\langle a_{1}\right\rangle_{s}\left[\left(\frac{\partial T_{0}}{\partial \eta_{k}}\right)^{2}+T_{0} \frac{\partial^{2} T_{0}}{\partial \eta_{k}^{2}}\right]\right\}, \\
= & \frac{1}{V^{(1)}+V^{(2)}}\left\{a_{0}^{(1)} V^{(1)}\left(c_{1}^{(1)}+1\right)\left[\frac{\partial^{2} T_{0}}{\partial \eta_{1}^{2}}+\frac{\partial^{2} T_{0}}{\partial \eta_{2}^{2}}+\frac{\partial^{2} T_{0}}{\partial \eta_{3}^{2}}\right]\right. \\
+ & a_{0}^{(2)} V^{(2)}\left(c_{1}^{(2)}+1\right)\left[\frac{\partial^{2} T_{0}}{\partial \eta_{1}^{2}}+\frac{\partial^{2} T_{0}}{\partial \eta_{2}^{2}}+\frac{\partial^{2} T_{0}}{\partial \eta_{3}^{2}}\right] \\
+ & a_{1}^{(1)} V^{(1)}\left(c_{1}^{(1)}+1\right)^{2}\left[\left(\frac{\partial T_{0}}{\partial \eta_{1}}\right)^{2}+\left(\frac{\partial T_{0}}{\partial \eta_{2}}\right)^{2}+\left(\frac{\partial T_{0}}{\partial \eta_{3}}\right)^{2}\right] \\
+ & a_{1}^{(2)} V^{(2)}\left[\left(c_{1}^{(2)}+1\right)^{2}+\frac{4\left[c_{2}^{(2)}\right]^{2}}{\left.5\left[r^{(1)} r^{(2)}\right]^{3}\right]}\left[\left(\frac{\partial T_{0}}{\partial \eta_{1}}\right)^{2}+\left(\frac{\partial T_{0}}{\partial \eta_{2}}\right)^{2}+\left(\frac{\partial T_{0}}{\partial \eta_{3}}\right)^{2}\right]\right. \\
+ & a_{1}^{(1)} V^{(1)}\left(c_{1}^{(1)}+1\right) T_{0}\left[\frac{\partial^{2} T_{0}}{\partial \eta_{1}^{2}}+\frac{\partial^{2} T_{0}}{\partial \eta_{2}^{2}}+\frac{\partial^{2} T_{0}}{\partial \eta_{3}^{2}}\right] \\
+ & a_{1}^{(2)} V^{(2)}\left(c_{1}^{(2)}+1\right) T_{0}\left[\frac{\partial^{2} T_{0}}{\partial \eta_{1}^{2}}+\frac{\partial^{2} T_{0}}{\partial \eta_{2}^{2}}+\frac{\partial^{2} T_{0}}{\partial \eta_{3}^{2}}\right] .
\end{aligned}
$$


Because the radius $r^{(3)}$ of the unit cell is taken to be of infinite length in the asymptotic model, $r^{(3)} \rightarrow \infty$, the single underlined term in (84) has vanished. The parameters $c_{1}^{(i)}$ and $c_{3}^{(i)}$ are also functions of $T_{0}$ and its derivatives. If we substitute $c_{1}^{(i)}$ and $c_{3}^{(i)}$ into (86), linearize the result and neglect all terms of higher order than in the effective heat equation, then we obtain the effective parameters.

- By comparing the factors $\frac{\partial^{2} T_{0}}{\partial \eta_{1}^{2}}$ in (86) we obtain

$$
\left\langle a_{0}\right\rangle_{s}=a_{0}^{(2)} \frac{a_{0}^{(1)}\left(\left[r^{(2)}\right]^{3}+2\left[r^{(1)}\right]^{3}\right)+2 a_{0}^{(2)} \hat{r}_{-}+2 a_{0}^{(1)} a_{0}^{(2)} \hat{b}_{1} \hat{r}_{-}}{a_{0}^{(1)} \hat{r}_{-}+a_{0}^{(2)}\left(2\left[r^{(2)}\right]^{3}+\left[r^{(1)}\right]^{3}\right)+a_{0}^{(1)} a_{0}^{(2)} \hat{b}_{1}\left(2\left[r^{(2)}\right]^{3}+\left[r^{(1)}\right]^{3}\right)},
$$

where $\hat{r}_{-}=\left[r^{(2)}\right]^{3}-\left[r^{(1)}\right]^{3}, \hat{b}_{1}=b_{1} / r^{(1)}$.

- If we compare the factors of either $T_{0} \frac{\partial^{2} T_{0}}{\partial \eta_{2}}$ or $\left(\frac{\partial T_{0}}{\partial \eta}\right)^{2}$ on both sides of (86) and substitute (87), then we obtain

$$
\begin{aligned}
\left\langle a_{1}\right\rangle_{s} & =-\left\{4\left(\left[a_{0}^{(1)}\right]^{2}+\left[a_{0}^{(2)}\right]^{2}\right) a_{1}^{(2)}\left[r^{(1)}\right]^{6}-\left(5\left[a_{0}^{(1)}\right]^{2}+20\left[a_{0}^{(2)}\right]^{2}\right) a_{1}^{(2)}\left[r^{(2)}\right]^{6}\right. \\
& -45 a_{1}^{(1)}\left[a_{0}^{(2)}\right]^{2}\left[r^{(1)} r^{(2)}\right]^{3}+\left(\left[a_{0}^{(1)}\right]^{2}+16\left[a_{0}^{(2)}\right]^{2}\right) a_{1}^{(2)}\left[r^{(1)} r^{(2)}\right]^{3} \\
& -a_{0}^{(1)} a_{0}^{(2)} a_{1}^{(2)}\left(8\left[r^{(1)}\right]^{6}+20\left[r^{(2)}\right]^{6}-28\left[r^{(1)} r^{(2)}\right]^{3}\right) \\
& +8\left(a_{0}^{(1)}\left[a_{0}^{(2)}\right]^{2}-\left[a_{0}^{(1)}\right]^{2} a_{0}^{(2)}\right) a_{1}^{(2)} \hat{b}_{1}\left[r^{(1)}\right]^{6} \\
& -\left(40 a_{0}^{(1)}\left[a_{0}^{(2)}\right]^{2}+20\left[a_{0}^{(1)}\right]^{2} a_{0}^{(2)}\right) a_{1}^{(2)} \hat{b}_{1}\left[r^{(2)}\right]^{6} \\
& +\left[a_{0}^{(1)} a_{0}^{(2)}\right]^{2} a_{1}^{(2)} \hat{b}_{1}^{2}\left(4\left[r^{(1)}\right]^{6}-20\left[r^{(2)}\right]^{6}+16\left[r^{(1)} r^{(2)}\right]^{3}\right) \\
& \left.+\left(32 a_{0}^{(1)}\left[a_{0}^{(2)}\right]^{2}+28\left[a_{0}^{(1)}\right]^{2} a_{0}^{(2)}\right) a_{1}^{(2)} \hat{b}_{1}\left[r^{(1)} r^{(2)}\right]^{3}\right\} \\
& /\left\{5 \left[a_{0}^{(1)}\left(\left[r^{(2)}\right]^{3}-\left[r^{(1)}\right]^{3}\right)+a_{0}^{(2)}\left(2\left[r^{(2)}\right]^{3}+\left[r^{(1)}\right]^{3}\right)\right.\right. \\
& \left.\left.+a_{0}^{(1)} a_{0}^{(2)} \hat{b}_{1}\left(2\left[r^{(2)}\right]^{3}+\left[r^{(1)}\right]^{3}\right)\right]^{2}\right\} .
\end{aligned}
$$

\subsection{Numerical examples}

The present sections presents some numerical examples for the effective thermal conductivities which are obtained by the application of the three-phase model. The first part of the numerical examples compares some special cases of our results to known results from the literature, and the second part analyzes the influence of temperature-dependence of the thermal conductivity of the constituents as well as the impact of the thermal resistance on the effective properties.

Comparison of the present solution for the effective thermal conductivity to known results. We consider a composite which consists of the inclusions $\Omega^{(1)}$ which are embedded in the matrix $\Omega^{(2)}$, and we assume no thermal resistance between the constituents, so that $b_{1}=0$. The effective thermal conductivity is taken to be temperature independent and defined as

$$
\langle k\rangle= \begin{cases}\left\langle a_{0}\right\rangle_{c} & \text { for cylindrical inclusions, } \\ \left\langle a_{0}\right\rangle_{s} & \text { for spherical inclusions, }\end{cases}
$$




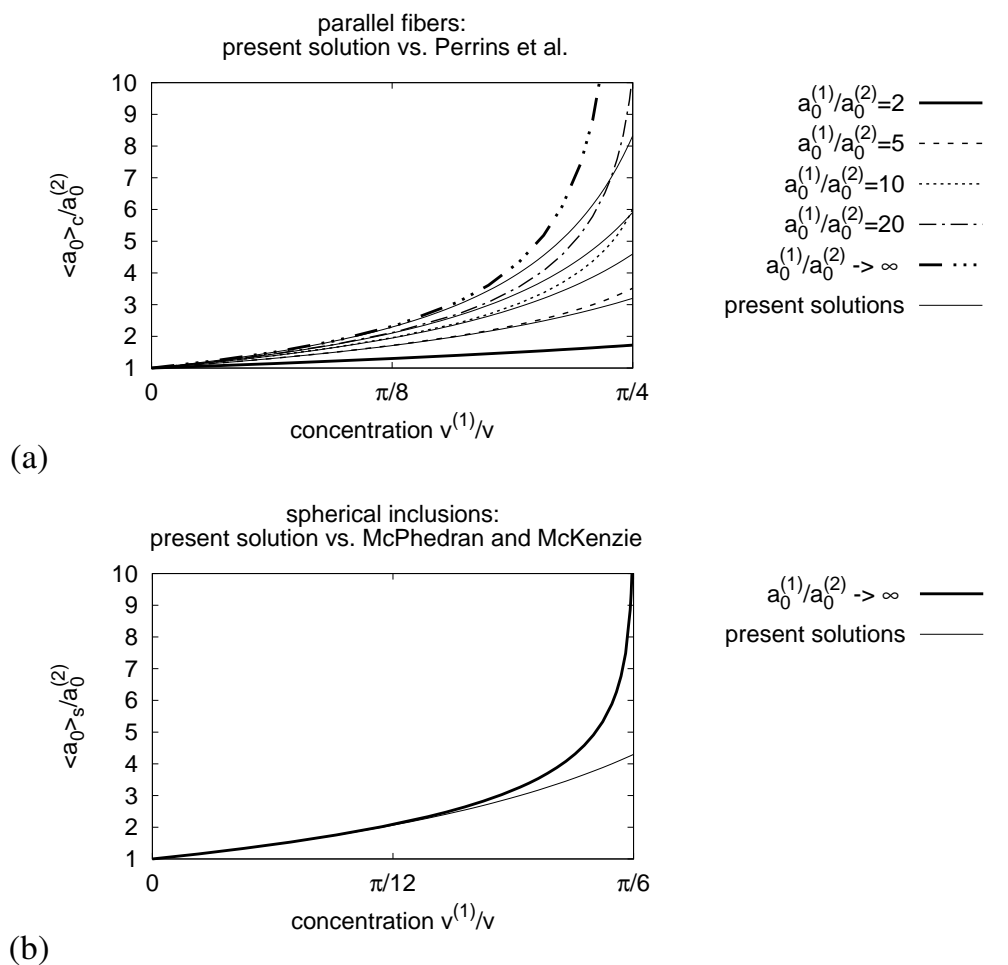

Figure 8: Panel (a): normalized effective thermal conductivity $\left\langle a_{0}\right\rangle_{c} / a_{0}^{(2)}$ versus the volume fraction $v^{(1)} / v$ of the cylindrical inclusions. The thin solid lines correspond to the present solution in (67), and all other lines to the results of Perrins et al. [50]. Panel (b): normalized effective thermal conductivity $\left\langle a_{0}\right\rangle_{c} / a_{0}^{(2)}$ versus the volume fraction $v^{(1)} / v$ of the spherical inclusions. The thin solid lines correspond to the present solution in (87), and the thick line to the results of McPhedran \& McKenzie [51].

where $\left\langle a_{0}\right\rangle_{c}$ is given in (67), and $\left\langle a_{0}\right\rangle_{s}$ is given in (87).

- Panel (a) of Fig. 8 shows the normalized effective thermal conductivity $\left\langle a_{0}\right\rangle_{c} / a_{0}^{(2)}$ versus the volume fraction $v^{(1)} / v$ of the inclusion. The thin solid lines correspond to the present solution in (67), and all other lines to the results of Perrins et al. [50].

- Panel (b) of Fig. 8 shows the normalized effective thermal conductivity $\left\langle a_{0}\right\rangle_{s} / a_{0}^{(2)}$ versus the volume fraction $v^{(1)} / v$ of the inclusion. The thin solid lines correspond to the present solution in (87), and the thick line to the results of McPhedran \& McKenzie [51].

For both the results for the cylindrical and spherical inclusions we find that there herein obtained results coincide well with the results from the literature in the case of small and intermediate volume fractions of the inclusion.

Effective temperature-dependent thermal conductivity for thermal resistance between the constituents. Consider a composite which consists of the inclusions $\Omega^{(1)}$ which are embedded in the matrix $\Omega^{(2)}$. The inclusion $\Omega^{(1)}$ is assumed to have the temperature-dependent thermal conductivity, $k^{(1)}=a_{0}^{(1)}+T_{0} a_{1}^{(1)}$, where $a_{0}^{(1)}=100 \mathrm{~W} \mathrm{~m}^{-1} \mathrm{~K}^{-1}$, and $T_{0}=293.15 \mathrm{~K}$ are chosen. We 
(a)
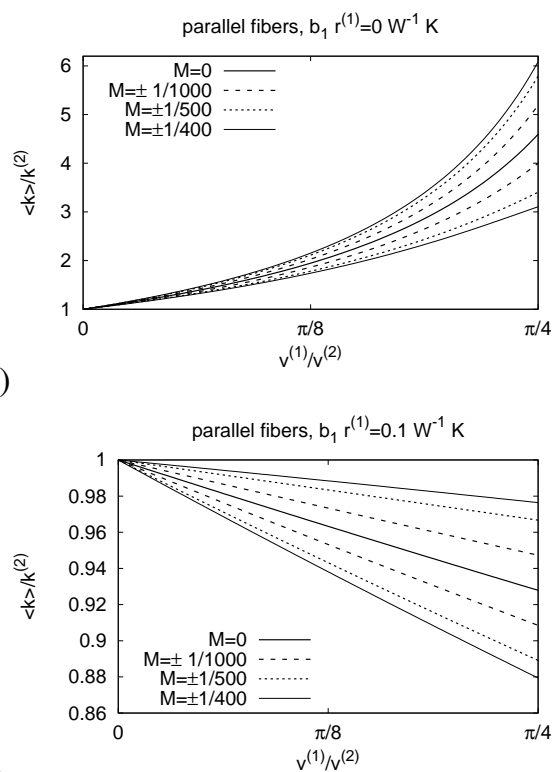

(c)

where $\left\langle a_{0}\right\rangle_{c}$ and $\left\langle a_{1}\right\rangle_{c}$ are given in (67) and (68), respectively, and $\left\langle a_{0}\right\rangle_{s}$ and $\left\langle a_{1}\right\rangle_{c}$ are given in (87) and (88), respectively.

$$
\langle k\rangle= \begin{cases}\left\langle a_{0}\right\rangle_{c}+T_{0}\left\langle a_{1}\right\rangle_{c} & \text { for cylindrical inclusions, } \\ \left\langle a_{0}\right\rangle_{s}+T_{0}\left\langle a_{1}\right\rangle_{s} & \text { for spherical inclusions, }\end{cases}
$$

(b)
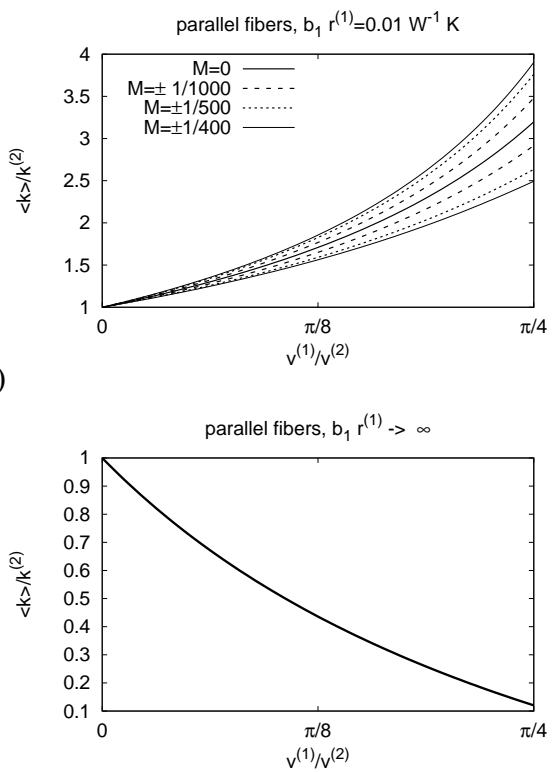

(d)

Figure 9: Normalized effective thermal conductivity $\langle k\rangle / k^{(2)}$ versus $v^{(1)} / v^{(2)}$ for cylindrical inclusions and different values for $M$, where $v^{(1)} / v^{(2)}=\pi / 4$ corresponds to the maximum volume fraction of the inclusions. The upper curves in a certain line style correspond the positive values for $M$, and the lower curves correspond to negative values for $M$.

- Figure 9 show the normalized effective thermal conductivity $\langle k\rangle / k^{(2)}$ versus $v^{(1)} / v^{(2)}$ for cylindrical inclusions and different values for $M$, where $v^{(1)} / v^{(2)}=\pi / 4$ corresponds to the maximum volume fraction of the inclusions. The upper curves in a certain line style correspond the positive values for $M$, and the lower curves correspond to negative values for $M$. Panel (a) shows the case of the absence of any thermal resistance between the constituents, panel (b) shows the case of $\hat{b}^{(1)}=b_{1} r^{(1)}=0.01 \mathrm{~W}^{-1} \mathrm{~K}$, panel (c) shows the case of $\hat{b}^{(1)}=0.1 \mathrm{~W}^{-1} \mathrm{~K}$, and panel (d) shows the case of $\hat{b}^{(1)} \rightarrow \infty$. Decreasing values for $M$ result in lower values for the thermal conductivity. If $\hat{b}_{1}$ becomes sufficiently large, then the 
(a)
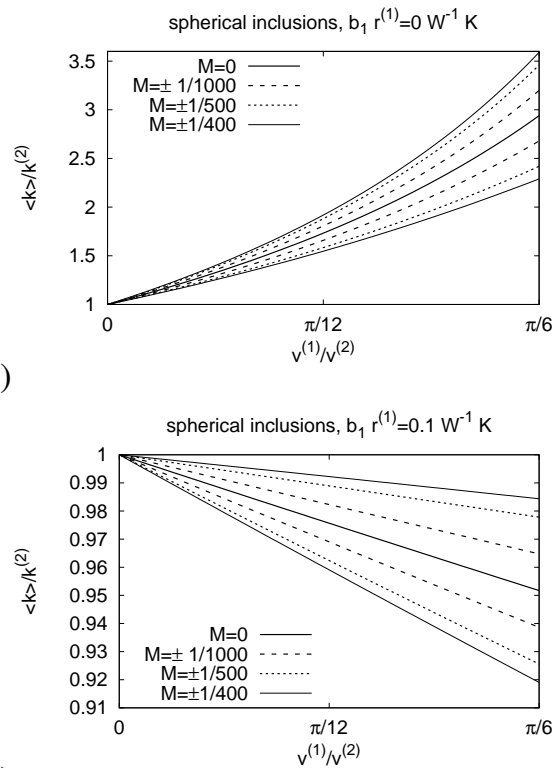

(c)

- Figure 10 show the normalized effective thermal conductivity $\langle k\rangle / k^{(2)}$ versus $v^{(1)} / v^{(2)}$ for
spherical inclusions and different values for $M$, where $v^{(1)} / v^{(2)}=\pi / 6$ corresponds to the
maximum volume fraction of the inclusions. As in the previous part, panel (a) shows the

- Figure 10 show the normalized effective thermal conductivity $\langle k\rangle / k^{(2)}$ versus $v^{(1)} / v^{(2)}$ for
spherical inclusions and different values for $M$, where $v^{(1)} / v^{(2)}=\pi / 6$ corresponds to the
maximum volume fraction of the inclusions. As in the previous part, panel (a) shows the

- Figure 10 show the normalized effective thermal conductivity $\langle k\rangle / k^{(2)}$ versus $v^{(1)} / v^{(2)}$ for
spherical inclusions and different values for $M$, where $v^{(1)} / v^{(2)}=\pi / 6$ corresponds to the
maximum volume fraction of the inclusions. As in the previous part, panel (a) shows the

effective properties decrease with increasing values of the inclusion. The case of $\hat{b}^{(1)} \rightarrow \infty$ describes a thermally insulated inclusion. In such case, the effective thermal conductivity depends on the geometry of the inclusions, but not on their thermal properties.

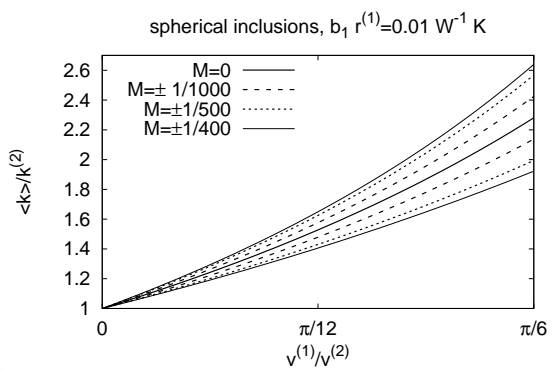

(b)

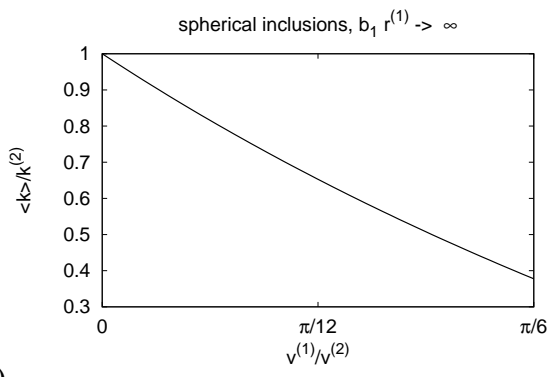

Figure 10: Normalized effective thermal conductivity $\langle k\rangle / k^{(2)}$ versus $v^{(1)} / v^{(2)}$ for spherical inclusions and different values for $M$, where $v^{(1)} / v^{(2)}=\pi / 6$ corresponds to the maximum volume fraction of the inclusions. The upper curves in a certain line style correspond the positive values for $M$, and the lower curves correspond to negative values for $M$.

case of the absence of any thermal resistance between the constituents, panel (b) shows the case of $\hat{b}^{(1)}=b_{1} r^{(1)}=0.01 \mathrm{~W}^{-1} \mathrm{~K}$, panel (c) shows the case of $\hat{b}^{(1)}=0.1 \mathrm{~W}^{-1} \mathrm{~K}$, and panel (d) shows the case of $\hat{b}^{(1)} \rightarrow \infty$.

\section{Conclusions}

In the present article we applied the AHM in order to obtain the effective thermal properties of a composite with a regular microstructure. These effective or homogenized thermal properties of the heterogeneous solid are obtained analytically in an explicit form. The thermal conductivity of the single constituents has been taken to be a polynomial in terms of the temperature, and the thermal resistance at the interface of the constituents has been taken to be nonlinear. The details of the heat diffusion model and the conjugate conditions are presented in Sec. 2. A general form of the homogenized heat equation is then derived in Sec. 3. This article then discusses some specific cases of the composite structure. 
In Sec. 4, we apply the AHM to derive a homogenized heat equation which is capable to describe the behavior of composites with inclusions of large volume fractions by the application of the well-known lubrication theory, see Christensen \& Lo [19] and Christensen [21]. The relatively simple case of a layered composite is also discussed to highlight some features of our results. In the numerical examples we illustrate both the impact of the different material parameters of the constituents and the interaction of the constituents on the effective thermal behavior. In these numerical examples, the finite difference method has been applied.

Different articles on the application of the AHM have shown, that the three-phase model is a useful method to derive the effective properties of periodic composites for the case of low volume-fractions of the inclusions. In the framework of this article we applied this method in Sec. 5, and we considered two cases for inclusions, parallel fibers and spherical inclusions. We also took into account thermal resistance at the common interfaces between the inclusions and the matrix. A special case of the our nonlinear homogenized solution, the linear case in absence of thermal resistance, has been compared to well-known results, i.e., to Perrins et al. [50] for cylindrical inclusions and to McPhedran \& McKenzie [51] for spherical inclusions. This comparison has shown that especially for small volume fractions of the inclusions our results coincidence the results from the literature. In the then following examples we studied the impact of temperature-dependent thermal conductivity and thermal resistance on the effective thermal properties of the composites.

For intermediate values of the volume fractions the results from the lubrication theory and the three-phase model can be combined, for example by the application of Padé approximants (see, for example $[52,53])$. In such approach, the results of the three-phase model will dominate when $\frac{v^{(1)}}{v}$ takes small values, and the results of the lubrication theory will dominate if $\frac{v^{(1)}}{v}$ becomes larger. For works which apply Padé approximants in the homogenization theory of heat transfer problems, we refer to the articles of Andrianov et al. [54] and Gałka et al. [16].

There herein present results find their applications in different fields of engineering, for example in the automotive industry, in the field of civil engineering, and also in military and space industry. Different studies are devoted to the development of encapsulated spherical phasechanging materials (PCM) in order to improve the thermal efficiency of buildings (see, for example, Didier et al. [55] and Krupa et al. [56]), especially for civil engineering applications in regions with rough climatic conditions such as the Arabian/Persian Gulf. Rockets and space vehicles are subjected to extreme thermal conditions, which motivates the development of new composite materials with the required thermal properties, for example by the use of a matrix components with stronger nonlinear thermal properties than the reinforcing component; see for example Fisher et al. [57] on ceramic composite thermal protection systems, Jenkins [58] on fibrous refractory composite insulation (FRCI) tiles applicable to the NASA Space Shuttle orbiter.

Together with the evaluation of the effective thermal properties the there herein presented asymptotic approaches allow to determine the distribution of the local temperature and flux fields on microlevel. This may be crucially important for the materials, which are used at rapidly changing temperatures under extreme conditions. 


\section{Appendix A. Simplification of the homogenized heat equation}

In order to derive the homogenized heat Eq. (36), we have applied

$$
\int_{-\frac{L}{2}}^{\frac{L}{2}} a_{k}^{(i)}\left(\frac{\partial^{2} T_{n-1}^{(i)}}{\partial \eta_{k} \partial \zeta_{k}}+\frac{\partial^{2} T_{n}^{(i)}}{\partial \zeta_{k}^{2}}\right) \mathrm{d} \zeta=0
$$

where $k=1,2$. This procedure removed all terms which contain the yet unknown corrections terms $T_{2}^{(i)}$ in (37). For details on the proof of (A.1), we refer to Appendix A1 in Topol [45].

\section{Appendix B. Finite difference method}

We apply finite difference method to approximate the solution to the homogenized equation (38) numerically. Therefore, let us apply the central difference scheme for the different forms of the derivatives of $T_{0}$ with respect to $\eta=\eta_{k}$ at the location $\eta=\eta_{p}$ and time $t=t_{q}$ which occur in the homogenized heat equation,

$$
\begin{aligned}
\left.\frac{\partial T_{0}(\eta, t)}{\partial \eta}\right|_{\eta_{p}, t_{q}} & \approx \frac{T_{p+1}^{q}-T_{p-1}^{q}}{2 \delta \eta} \\
\left.\left(\frac{\partial T_{0}(\eta, t)}{\partial \eta}\right)^{2}\right|_{\eta_{p}, t_{q}} & \approx \frac{\left[T_{p+1}^{q}\right]^{2}-2 T_{p+1}^{q} T_{p-1}^{q}+\left[T_{p-1}^{q}\right]^{2}}{4 \delta \eta^{2}}, \\
\left.\frac{\partial^{2} T_{0}(\eta, t)}{\partial \eta^{2}}\right|_{\eta_{p}, t_{q}} & \approx \frac{T_{p+1}^{q}-2 T_{p}^{q}+T_{p-1}^{q}}{\delta \eta^{2}},
\end{aligned}
$$

where $\delta \eta=\eta_{p+1}-\eta_{p}$ is a location step. The subscript $p$ in $T_{p}^{q}$ refers to the location $\eta_{p}$, and the superscript $q$ refers to the time $t_{q}$, i.e., $T_{p+1}^{q}=T_{0}\left(\eta_{p}+\delta \eta, t_{q}\right), T_{p}^{q}=T_{0}\left(\eta_{p}, t_{q}\right)$, and $T_{p-1}^{q}=$ $T_{0}\left(\eta_{p}-\delta \eta, t_{q}\right)$. Let us now also apply the forward difference method to approximate the derivative of $T_{0}$ with respect to $t$ at the location $\eta=\eta_{p}$ and time $t=t_{q}$,

$$
\left.\frac{\partial T_{0}(\eta, t)}{\partial t}\right|_{\eta_{p}, t_{q}} \approx \frac{T_{p}^{q+1}-T_{p}^{q}}{\delta t}
$$

where $\delta t=t_{q+1}-t_{q}$ is time step, $T_{p}^{q+1}=T_{0}\left(\eta_{p}, t_{q}\right)$, and $T_{p}^{q+1}=T_{0}\left(\eta_{p}, t_{q}+\delta t\right)$. If the substitute the finite difference approximations (B.1) and (B.2) into the homogenized heat Eq. (38), we derive the following systems of equations,

$$
\begin{aligned}
\frac{T_{p}^{q+1}-T_{p}^{q}}{\delta t} & =\left\langle\bar{a}_{0}\right\rangle \frac{T_{p+1}^{q}-2 T_{p}^{q}+T_{p-1}^{q}}{\delta \eta^{2}}+\left\langle\bar{a}_{1}\right\rangle\left[\frac{\left[T_{p+1}^{q}\right]^{2}-2 T_{p+1}^{q} T_{p-1}^{q}+\left[T_{p-1}^{q}\right]^{2}}{4 \delta \eta^{2}}\right. \\
& \left.+T_{p}^{q} \frac{T_{p+1}^{q}-2 T_{p}^{q}+T_{p-1}^{q}}{\delta \eta^{2}}\right]_{34}
\end{aligned}
$$


where $\left\langle\bar{a}_{i}\right\rangle=\left\langle a_{i}\right\rangle /\left\langle\rho_{p}\right\rangle$. If we now rewrite (B.3) in the following way

$$
\begin{aligned}
T_{p}^{q+1} & =\left\{\left\langle\bar{a}_{0}\right\rangle\left[\frac{T_{p+1}^{q}-2 T_{p}^{q}+T_{p-1}^{q}}{\delta \eta^{2}}\right]+\left\langle\bar{a}_{1}\right\rangle\left[\frac{\left[T_{p+1}^{q}\right]^{2}-2 T_{p+1}^{q} T_{p-1}^{q}+\left[T_{p-1}^{q}\right]^{2}}{4 \delta \eta^{2}}\right.\right. \\
& \left.\left.+T_{p}^{q} \frac{T_{p+1}^{q}-2 T_{p}^{q}+T_{p-1}^{q}}{\delta \eta^{2}}\right]\right\} \delta t+T_{p}^{q},
\end{aligned}
$$

In (80) we have applied the abbreviations

$$
\begin{aligned}
\frac{\partial^{2} T_{0}}{\partial \eta_{1}^{2}}+\frac{\partial^{2} T_{1}^{(i)}}{\partial \eta_{1} \partial \zeta_{1}} & =\frac{\partial^{2} T_{0}}{\partial \eta_{1}^{2}}+\cos \phi \sin \theta \frac{\partial^{2} \hat{T}_{1}^{(1)}}{\partial \eta_{1} \partial r} \\
& -\frac{\sin \phi}{r \sin \theta} \frac{\partial^{2} \hat{T}_{1}^{(1)}}{\partial \eta_{1} \partial \phi}+\frac{\cos \phi \cos \theta}{r} \frac{\partial^{2} \hat{T}_{1}^{(1)}}{\partial \eta_{1} \partial \theta}, \\
\frac{\partial T_{0}}{\partial \eta_{1}}+\frac{\partial T_{1}^{(i)}}{\partial \zeta_{1}} & =\frac{\partial T_{0}}{\partial \eta_{1}}+\cos \phi \sin \theta \frac{\partial \hat{T}_{1}^{(1)}}{\partial r}-\frac{\sin \phi}{r \sin \theta} \frac{\partial \hat{T}_{1}^{(1)}}{\partial \phi} \\
& +\frac{\cos \phi \cos \theta}{r} \frac{\partial \hat{T}_{1}^{(1)}}{\partial \theta},
\end{aligned}
$$


and

$$
\begin{aligned}
\frac{\partial^{2} T_{0}}{\partial \eta_{2}^{2}}+\frac{\partial^{2} \hat{T}_{1}^{(i)}}{\partial \eta_{2} \partial \zeta_{2}} & =\frac{\partial^{2} T_{0}}{\partial \eta_{2}^{2}}+\sin \phi \sin \theta \frac{\partial^{2} \hat{T}_{1}^{(1)}}{\partial \eta_{2} \partial r} \\
& +\frac{\cos \phi}{r \sin \theta} \frac{\partial^{2} \hat{T}_{1}^{(1)}}{\partial \eta_{2} \partial \phi}+\frac{\sin \phi \cos \theta}{r} \frac{\partial^{2} \hat{T}_{1}^{(1)}}{\partial \eta_{2} \partial \theta}, \\
\frac{\partial T_{0}}{\partial \eta_{2}}+\frac{\partial \bar{T}_{1}^{(i)}}{\partial \zeta_{2}} & =\frac{\partial T_{0}}{\partial \eta_{2}}+\sin \phi \sin \theta \frac{\partial \hat{T}_{1}^{(1)}}{\partial r}+\frac{\cos \phi}{r \sin \theta} \frac{\partial \hat{T}_{1}^{(1)}}{\partial \phi} \\
& +\frac{\sin \phi \cos \theta}{r} \frac{\partial \hat{T}_{1}^{(1)}}{\partial \theta},
\end{aligned}
$$

and

$$
\begin{aligned}
& \frac{\partial^{2} T_{0}}{\partial \eta_{3}^{2}}+\frac{\partial^{2} \hat{T}_{1}^{(i)}}{\partial \eta_{3} \partial \zeta_{3}}=\frac{\partial^{2} T_{0}}{\partial \eta_{3}^{2}}+\cos \theta \frac{\partial^{2} T_{1}^{(1)}}{\partial \eta_{3} \partial r}-\frac{\sin \theta}{r} \frac{\partial^{2} T_{0}}{\partial \eta_{3} \partial \theta} \\
& \frac{\partial T_{0}}{\partial \eta_{3}}+\frac{\partial \bar{T}_{1}^{(i)}}{\partial \zeta_{3}}=\frac{\partial T_{0}}{\partial \eta_{3}}+\cos \theta \frac{\partial T_{1}^{(1)}}{\partial r}-\frac{\sin \theta}{r} \frac{\partial T_{1}^{(1)}}{\partial \theta}
\end{aligned}
$$

\section{Acknowledgements}

The authors are grateful to the anonymous reviewers for their valuable comments and suggestions, which helped to improve the paper. This work was supported by a Qatar University Internal Grant (QUUG-CAM-CAM-15/16-3) for H. Topol. This work has received funding from the European Union's Horizon 2020 research and innovation program under the Marie SklodowskaCurie grant agreement no. 655177 for V.V. Danishevskyy.

\section{References}

[1] A. V. Hershey, The elasticity of an isotropic aggregate of anisotropic cubic crystals, J. Appl. Mech. 21 (1954) 236-240.

[2] R. Hill, A self-consistent mechanics of composite materials, J. Mech. Phys. Solids 13 (1965) $213-222$.

[3] E. H. Kerner, The elastic and thermoelastic properties of composite media, Proc. Phys. Soc. B 69 (1956) 808-813.

[4] E. Kröner, Berechnung der elastischen Konstanten des Vielkristalls aus den Konstanten des Einkristalls, Z. Physik 151 (1958) 504-518.

[5] J. B. Keller, A theorem on the conductivity of a composite medium, J. Math. Phys. 5 (1964) 548-549.

[6] C. van der Poel, On the rheology of concentrated dispersions, Rheol. Acta 1 (1958) 198-211.

[7] I. Özdemir, W. A. M. Brekelmans, M. G. D. Geers, Computational homogenization for heat conduction in heterogeneous solids, Int. J. Numer. Meth. Engng. 73 (2008) 185-204.

[8] M. G. D. Geers, V. G. Kouznetsova, W. A. M. Brekelmans, Multi-scale computational homogenization: Trends and challenges, J. Comput. Appl. Math. 234 (2010) 2175-2182.

[9] A. Bensoussan, J.-L. Lions, G. Papanicolaou, Asymptotic Analysis for Periodic Structures, North-Holland, 1978.

[10] G. Panasenko, Multi-scale Modelling for Structures and Composites, Springer, 2005.

[11] I. V. Andrianov, V. I. Bolshakov, V. V. Danishevs'kyy, D. Weichert, Higher order asymptotic homogenization and wave propagation in periodic composite materials, Proc. R. Soc. A 464 (2008) 1181-1201.

[12] W. J. Parnell, I. D. Abrahams, Homogenization for wave propagation in periodic fibre-reinforced media with complex microstructure. I-Theory, J. Mech. Phys. Solids 56 (2008) 2521-2540.

[13] G. Allaire, Homogenization and two-scale convergence, SIAM J. Math. Anal. 23 (1992) 1482-1518.

[14] H. W. Zhang, S. Zhang, J. Y. Bi, B. A. Schrefler, Thermo-mechanical analysis of periodic multiphase materials by a multiscale asymptotic homogenization approach, Int. J. Numer. Meth. Engng. 69 (2007) 87-113. 
[15] J. J. Telega, S. Tokarzewski, A. Gałka, Effective conductivity of nonlinear two-phase media: Homogenization and two-point Padé approximants, Acta Appl. Math. 61 (2000) 295-315.

[16] A. Gałka, J. J. Telega, S. Tokarzewski, Heat equation with temperature-dependent conductivity coefficients and macroscopic properties of microheterogeneous media, Math. Comput. Model 33 (2001) 927-942.

[17] G. Allaire, Z. Habibi, Homogenization of a conductive, convective, and radiative heat transfer problem in a heterogeneous domain, SIAM J. Math. Anal. 45 (2013) 1136-1178.

[18] Z. Yang, J. Cui, Z. Wang, Y. Zhang, Multiscale computational method for nonstationary integrated heat transfer problem in periodic porous materials, Numer. Meth. Part. D. E. 32 (2016) 510-530.

[19] R. M. Christensen, K. H. Lo, Solutions for effective shear properties in three phase sphere and cylinder models, J. Mech. Phys. Solids 27 (1979) 315-330.

[20] I. V. Andrianov, V. V. Danishevs'kyy, A. L. Kalamkarov, Asymptotic justification of three-phase composite model, Compos. Struct. 77 (2007) 395-404.

[21] R. M. Christensen, Mechanics of Composite Materials, Dover Publications, 2005.

[22] A. L. Kalamkarov, I. V. Andrianov, V. V. Danishevs'kyy, Asymptotic homogenization of composite materials and structures, Appl. Mech. Rev. 62 (2009) 030802 (20 pages).

[23] P. L. Kapitza, The study of heat transfer in helium II, J. Phys. (USSR) 4 (1941) 181-210.

[24] M. Karkri, M. Lachheb, F. Albouchi, S. Ben Nasrallah, I. Krupa, Thermal properties of smart microencapsulated paraffin/plaster composites for the thermal regulation of buildings, Energy Build. 88 (2015) 183-192.

[25] M. Karkri, M. Lachheb, Z. Nógellová, B. Boh, B. Sumiga, M. A. AlMaadeed, A. Fethi, I. Krupa, Thermal properties of phase-change materials based on high-density polyethylene filled with micro-encapsulated paraffin wax for thermalenergy storage, Energy Build. 88 (2015) 144-152.

[26] H. L. Quang, G. Bonnet, Q.-C. He, Size-dependent eshelby tensor fields and effective conductivity of composites made of anisotropic phases with highly conducting imperfect interfaces, Phys. Rev. B 81 (2010) 064203.

[27] H. L. Quang, G. Bonnet, Q.-C. He, Eshelby's tensor fields and effective conductivity of composites made of anisotropic phases with Kapitza's interface thermal resistance, Philos. Mag. 91 (2011) 3358-3392.

[28] H. L. Quang, D. C. Pham, G. Bonnet, Q.-C. He, Estimations of the effective conductivity of anisotropic multiphase composites with imperfect interfaces, Int. J. Heat Mass Tran. 58 (2013) 175-187.

[29] I. V. Andrianov, V. I. Bolshakov, V. V. Danishevs'kyy, D. Weichert, Asymptotic study of imperfect interfaces in conduction through a granular composite material, Proc. R. Soc. A. 466 (2010) 2707-2725.

[30] G. C. Papanicolaou, M. V. Michalopoulou, N. K. Anifantis, Thermal stresses in fibrous composites incorporating hybrid interphase regions, Compos. Sci. Technol. 62 (2002) 1881-1894.

[31] N. Lombardo, Effect of an inhomogeneous interphase on the thermal expansion coefficient of a particulate composite, Compos. Sci. Technol. 68 (2005) 2118-2128.

[32] J. Lienemann, A. Yousefi, J. G. Korvink, Nonlinear heat transfer modeling, Lect. Notes Comp. Sci. 45 (2005) 327-331.

[33] J. E. Hatch, Aluminum: Properties and Physical Metallurgy, American Society for Metals, 1984.

[34] J. Hristov, An approximate analytical (integral-balance) solution to a nonlinear heat diffusion equation, Therm. Sci. 19 (2015) 723-733.

[35] M. Goland, E. Reissner, The stresses in cemented joints, J. Appl. Mech. 11 (1944) 17-27.

[36] I. V. Andrianov, V. V. Danishevs'kyy, H. Topol, D. Weichert, Homogenization of a 1D nonlinear dynamical problem for periodic composites, Z. angew. Math. Mech. 91 (2011) 523-534.

[37] N. S. Bakhvalov, G. P. Panasenko, Homogenization: Averaging Processes in Periodic Media: Mathematical Problems in the Mechanics of Composite Material, Kluver Academic, 1989.

[38] K. D. Cherednichenko, V. P. Smyshlyaev, V. V. Zhikov, Non-local homogenized limits for composite media with highly anisotropic periodic fibres, Proc. R. Soc. Edin. A 136 (2006) 87-114.

[39] G. A. Baker, P. Graves-Morris, Padé Approximants, Cambridge University Press, 1996.

[40] L. Berlyand, A. G. Kolpakov, A. Novikov, Introduction to the Network Approximation Method for Materials Modeling, Cambridge University Press, 2013.

[41] I. V. Andrianov, V. V. Danishevs'kyy, J. Awrejcewicz, Shear waves dispersion in cylindrically structured cancellous viscoelastic bones, in: J. Awrejcewicz (Ed.), Applied Non-Linear Dynamical Systems - Springer Proceedings in Mathematics and Statistics 93, Springer International Publishing, 2014, pp. 85-101.

[42] N. A. Frankel, A. Acrivos, On the viscosity of a concentrated suspension of solid spheres, Chem. Eng. Sci. 22 (1967) 847-853.

[43] A. B. Tayler, Mathematical Models in Applied Mechanics, Clarendon Press, 2001.

[44] I. V. Andrianov, V. V. Danishevs'kyy, D. Weichert, Asymptotic determination of effective elastic properties of composite materials with fibrous square-shaped inclusions, Eur. J. Mech. A Solids 21 (2002) 1019-1036.

[45] H. Topol, Acoustic and mechanical properties of viscoelastic, linear elastic, and nonlinear elastic composites, Ph.D. thesis, RWTH Aachen University (2012).

[46] G. D. Smith, Numerical Solution of Partial Differential Equations: Finite Difference Methods, Oxford Univerity 
Press, 1986.

[47] Nuclear Power Technology Development Section, International Atomic Energy Agency - Vienna, Austria, Thermophysical properties of materials for nuclear engineering: A tutorial and collection of data (2008).

[48] A. N. Guz, Y. N. Nemish, Perturbation of boundary shape in continuum mechanics, Soviet Appl. Mech. 23 (1987) 799-822.

[49] A. L. Kalamkarov, I. V. Andrianov, G. A. Starushenko, Three-phase model for a composite material with cylindrical circular inclusions. Part I: Application of the boundary shape perturbation method, Int. J. Eng. Sci. 78 (2014) 154177.

[50] W. T. Perrins, D. R. McKenzie, R. C. McPhedran, Transport properties of regular arrays of cylinders, Proc. R. Soc. A. 369 (1979) 830-834.

[51] R. C. McPhedran, D. R. McKenzie, The conductivity of lattices of spheres. I. The simple cubic lattice, Proc. R. Soc. Lond. A 359 (1978) 45-63.

[52] I. V. Andrianov, L. I. Manevitch, Asymptotology - Ideas, Methods, and Applications, Kluwer Academic Publishers, 2002.

[53] J. Awrejcewicz, I. V. Andrianov, L. I. Manevitch, Asymptotic Approaches in Nonlinear Dynamics, Springer, 1998.

[54] I. V. Andrianov, G. A. Starushenko, V. Danishevs'kyy, S. Tokarzewski, Homogenization procedure and Padé approximants for effective heat conductivity of composite materials with cylindrical inclusions having square crosssection, Proc. R. Soc. Lond. A 455 (1999) 3401-3413.

[55] G. Didier, M. Karkri, M. A. AlMaadeed, I. Krupa, A new experimental device and inverse method to characterize thermal properties of composite phase change materials, Compos. Struct. 133 (2015) 1149-1159.

[56] I. Krupa, Z. Nógellová, Z. Špitalský, I. Janigová, B. Boh, B. Sumiga, A. Kleinovába, M. Karkri, M. A. AlMaadeed, Phase change materials based on high-density polyethylene filled with microencapsulated paraffin wax, Energy Convers. Manage. 87 (2014) 400-409.

[57] R. E. Fisher, C. V. Burkland, W. E. Bustamante, Ceramic composite thermal protection systems, in: W. Smothers (Ed.), Proceedings of the 9th Annual Conference on Composites and Advanced Ceramic Materials: Ceramic Engineering and Science Proceedings, Volume 6, John Wiley \& Sons, 1985.

[58] D. R. Jenkins, Space Shuttle: The History of the National Space Transportation System, Voyager Press, 2007. 